Article

\title{
Density, Viscosity and Free Energy of Activation for Viscous Flow of Monoethanol Amine (1) $+\mathrm{H}_{2} \mathrm{O}(2)+$ $\mathrm{CO}_{2}$ (3) Mixtures
}

\author{
Sumudu S. Karunarathne ${ }^{\circledR}$, Dag A. Eimer and Lars E. Øi * \\ Faculty of Technology, Natural Sciences and Maritime Studies, University of South-Eastern Norway, \\ Kjølnes Ring 56, 3901 Porsgrunn, Norway; sumuduunimrt@gmail.com (S.S.K.); dag.a.eimer@usn.no (D.A.E.) \\ * Correspondence: lars.oi@usn.no; Tel.: +47-35575141
}

Received: 4 December 2019; Accepted: 7 January 2020; Published: 9 January 2020

\begin{abstract}
Densities and viscosities of aqueous monoethanol amine (MEA) and $\mathrm{CO}_{2}$-loaded aqueous MEA are highly relevant in engineering calculations to perform process design and simulations. Density and viscosity of the aqueous MEA were measured in the temperature range of $293.15 \mathrm{~K}$ to $363.15 \mathrm{~K}$ with MEA mass fractions ranging from 0.3 to 1.0. Densities of the aqueous MEA were fitted for a density correlation. Eyring's viscosity model based on absolute rate theory was adopted to determine the excess free energy of activation for viscous flow of aqueous MEA mixtures and was correlated by a Redlich-Kister polynomial. Densities and viscosities of $\mathrm{CO}_{2}$-loaded MEA solutions were measured in the temperature range of $293.15 \mathrm{~K}$ to $353.15 \mathrm{~K}$ with MEA mass fractions of $0.3,0.4$ and 0.5. The density correlation used to correlate aqueous MEA was modified to fit $\mathrm{CO}_{2}$-loaded density data. The free energy of activation for viscous flow for $\mathrm{CO}_{2}$-loaded aqueous MEA solutions was determined by Eyring's viscosity model and a correlation was proposed to represent free energy of activation for viscous flow and viscosity. This can be used to evaluate quantitative and qualitative properties in the MEA $+\mathrm{H}_{2} \mathrm{O}+\mathrm{CO}_{2}$ mixture.
\end{abstract}

Keywords: density; viscosity; Eyring's viscosity model; MEA

\section{Introduction}

Post-combustion $\mathrm{CO}_{2}$ capture (PCC) using absorption and desorption has gained great attention in the last decades and several amines have been investigated for their absorption efficiency. In acid gas treatment, monoethanol amine (MEA, IUPAC name: 2-aminoethanol) has been used since 1930 [1]. It is the benchmark amine for the evaluation of other amines in $\mathrm{CO}_{2}$ capture performance considering absorption efficiency, reaction rates, energy demand and corrosion resistance. A blend of $30 \%$ MEA with $70 \% \mathrm{H}_{2} \mathrm{O}$ by mass is a standard in PCC. Higher reaction rates of MEA with $\mathrm{CO}_{2}$ compared to secondary and tertiary amines enables optimization of the dimensions and operational parameters of the absorber column. MEA's low-absorption capacity and high-energy demand for desorption and poor corrosion resistance are arguments against the use of MEA at the commercial scale [2,3].

Density and viscosity of pure, aqueous and $\mathrm{CO}_{2}$-loaded aqueous MEA have been studied and reported in the literature under different temperatures, MEA concentrations and $\mathrm{CO}_{2}$ loadings [1,4-12]. These data are vital for development of empirical correlations that are useful in various aspects of process equipment design and process simulations. Density is important to determine the physical solubility of $\mathrm{CO}_{2}$ in solvent, the solvent kinetics and mass transfer. Viscosity is frequently used in the modified Stoke-Einstein equation to estimate diffusivity that is necessary for calculating mass transfer and kinetic properties [13,14]. Many references are available for data of aqueous MEA solutions under different MEA concentrations and temperatures. There is a lack of measured data for physical properties 
of $\mathrm{CO}_{2}$-loaded solutions at different $\mathrm{CO}_{2}$ loadings under different MEA concentrations. In order to reduce the unmeasured regions and to check the validity of measured data, further experimental studies are necessary.

Amundsen, Øi and Eimer [6] have used the McAllister three-body model [15] to represent the kinematic viscosity. Weiland, Dingman, Cronin and Browning [9] and Hartono, Mba and Svendsen [10] measured both density and viscosity of $\mathrm{CO}_{2}$-loaded aqueous MEA solutions and proposed correlations to fit the data. The approach of using a Redlich-Kister [16] type polynomial to predict excess volume for the aqueous MEA solutions in the density correlations is widely used. A similar approach to correlate excess viscosity is adopted by Islam, et al. [17] for aqueous MEA.

In this work, density and viscosity of aqueous MEA and $\mathrm{CO}_{2}$-loaded aqueous MEA were measured. The density correlation proposed by Aronu, Hartono and Svendsen [14] for the aqueous amino acid salt and amine amino acid salt solutions was used to correlate the density data of aqueous MEA. The same correlation was modified to predict the density of $\mathrm{CO}_{2}$-loaded aqueous MEA solutions. The parameters of the correlations were found through a regression analysis. Eyring's viscosity model [18] was used to calculate the free energy of activation for viscous flow of the aqueous MEA solutions and parameters of the Redlich-Kister type polynomial were estimated by regression. For the viscosity of $\mathrm{CO}_{2}$-loaded solutions, the difference of activation energy between $\mathrm{CO}_{2}$-loaded aqueous MEA and aqueous MEA solutions were calculated, and a correlation was proposed.

\section{Materials and Methods}

\subsection{Sample Preparation and $\mathrm{CO}_{2}$ Loading Analysis}

Descriptions of materials used in this study are given in Table 1. The Milli-Q water (resistivity 18.2 $\mathrm{M} \Omega \cdot \mathrm{cm}$ ) was degassed using a rotary evaporator connected to a vacuum pump to remove any dissolved gasses. The weights of liquids were measured through an electronic balance from Mettler Toledo (XS403S, Mettler Toledo, Greifensee, Switzerland) with a resolution of $1 \mathrm{mg}$. Aqueous MEA solutions with MEA to $\mathrm{H}_{2} \mathrm{O}$ mass ratio $w_{1}=0.3,0.4$, and 0.5 were prepared and fully loaded by bubbling $\mathrm{CO}_{2}$ through the solution until the $\mathrm{pH}$ become steady over time. Then different $\mathrm{CO}_{2}$-loaded solutions were prepared by diluting them with corresponding aqueous MEA. The amount of $\mathrm{CO}_{2}$ loaded to the aqueous MEA was determined by a titration method in which $\mathrm{CO}_{2}$ was fixed as $\mathrm{BaCO}_{3}$ via adding $50 \mathrm{~mL}$ of each $0.1 \mathrm{M} \mathrm{NaOH}$ and $0.3 \mathrm{M} \mathrm{BaCl}_{2}$ to $0.1-0.2 \mathrm{~g}$ of $\mathrm{CO}_{2}$ loaded solution. All the samples were boiled for approximately $10 \mathrm{~min}$ to ensure the completion of chemical reactions and were cooled until the temperature reaches the room conditions. Eventually, $\mathrm{BaCO}_{3}$ was separated by filtering using a hydrophilic polypropylene membrane filter $(47 \mathrm{~mm}, 0.45 \mu \mathrm{m})$. The filtered $\mathrm{BaCO}_{3}$ was put into $100 \mathrm{~mL}$ of distilled water and titrated with $0.1 \mathrm{M} \mathrm{HCl} \mathrm{until} \mathrm{the} \mathrm{solution} \mathrm{reached} \mathrm{pH}$ of 2 . Meanwhile, care needed to be taken to make sure all the $\mathrm{BaCO}_{3}$ was dissolved during the titration. Then, the sample was boiled and cooled again before it was titrated with $0.1 \mathrm{M} \mathrm{NaOH}$. Finally, the MEA concentration of mixtures was determined by titrating $1 \mathrm{~g}$ of $\mathrm{CO}_{2}$-loaded solution with $1 \mathrm{M} \mathrm{HCl}$.

Table 1. Materials used in this study ${ }^{a, b}$.

\begin{tabular}{ccccc}
\hline Chemical Name & CAS Reg. No. & Mole Fraction Purity & Source & Purification \\
\hline monoethanol amine $(\mathrm{MEA})$ & $141-43-5$ & $\geq 0.995\left(\mathrm{GC}^{\mathrm{b}}\right)$ & Sigma-Aldrich & no \\
carbon dioxide $\left(\mathrm{CO}_{2}\right)$ & $124-38-9$ & 0.99999 & AGA Norge AS & no \\
nitrogen $\left(\mathrm{N}_{2}\right)$ & $7727-37-9$ & 0.99999 & AGA Norge AS & no \\
sodium hydroxide $(\mathrm{NaOH})$ & $1310-73-2$ & - & Merck KGaA & no \\
hydrochloric acid $(\mathrm{HCl})$ & $7647-01-0$ & - & Merck KGaA & no \\
barium chloride dihydrate & $10326-27-9$ & $\geq 0.99$ & Merck KGaA & no \\
$\left(\mathrm{BaCl}_{2} \cdot 2 \mathrm{H}_{2} \mathrm{O}\right)$ & & & & \\
\hline
\end{tabular}

\footnotetext{
${ }^{a}$ As mentioned by the supplier. ${ }^{b}$ Gas-liquid chromatography.
} 


\subsection{Density Measurements}

The density of aqueous MEA and $\mathrm{CO}_{2}$-loaded aqueous MEA was measured by a DMA 4500 density meter from Anton Paar (Graz, Austria). The standard calibration procedure for DMA 4500 was performed using degassed water and air at $293.15 \mathrm{~K}$ occasionally, while density checks were performed frequently to check the validity of the previous calibration at $293.15 \mathrm{~K}$. Samples were inserted into the U-tube with care to prevent the presence of air bubbles in the tube. Measurements were performed using a separate sample at each temperature and composition. A cleaning and drying process of the U-tube was performed every time before a new sample was introduced. Density measurements were performed for the aqueous MEA of $w_{1}$ from 0.3 to 1 for the temperature range from $293.15 \mathrm{~K}$ to $363.15 \mathrm{~K}$ and $\mathrm{CO}_{2}$-loaded aqueous MEA of $w_{1}=0.3,0.4$ and 0.5 under different $\mathrm{CO}_{2}$ loading for the temperature range from $293.15 \mathrm{~K}$ to $353.15 \mathrm{~K}$. Final density data are presented as an average of three density measurements at each temperature and composition.

\subsection{Viscosity Measurements}

The dynamic viscosity was measured using a double-gap concentric rheometer Physica MCR 101 from Anton Paar (pressure cell XL DG35.12/PR; measuring cell serial number 80462200) (Graz, Austria). The standard viscosity solution S3S from Paragon Scientific Ltd. was used to calibrate the rheometer at different temperatures. The calibration and the measurement were done by using $7 \mathrm{~mL}$ of liquid volume under the shear rate $(\gamma)$ of $1000 \mathrm{~s}^{-1}$. Having compared with the reference viscosity data, measured viscosities of standard viscosity solution were used to determine the viscosity deviations at different temperatures. For temperatures where the supplier did not specify any reference viscosities, expected viscosity deviations were obtained via interpolation. A temperature controlling system with standard temperature uncertainty of $\pm 0.03 \mathrm{~K}$ is equipped with the rheometer. An external cooling system of Anton Paar Viscotherm VT2 (Graz, Austria) with standard temperature uncertainty of $\pm 0.02 \mathrm{~K}$ is employed for better temperature control in the range from $293.15 \mathrm{~K}$ to $303.15 \mathrm{~K}$. The solution in the rheometer was pressured by $\mathrm{N}_{2}$ gas ( $p=4$ bar) to minimize the possible release of MEA and $\mathrm{CO}_{2}$ into the gas phase. Viscosity measurements were performed for the aqueous MEA of $w_{1}$ from 0.3 to 1 in the temperature range from $293.15-363.15 \mathrm{~K}$ and $\mathrm{CO}_{2}$-loaded aqueous MEA with $w_{1}=0.3,0.4$ and 0.5 under different $\mathrm{CO}_{2}$ loadings for the temperature range from $293.15 \mathrm{~K}$ to $353.15 \mathrm{~K}$. The viscosity data presented in this study are the averaged measurements for minimum of three different measurements.

\section{Experimental Uncertainty}

The Guide to the expression of Uncertainty in Measurement (GUM) $[19,20]$ approach was adopted for the uncertainty evaluations using the mathematical models defined for the instruments for density and viscosity measurements. Several uncertainty sources including purity of MEA, weight measurements, repeatability, $\mathrm{CO}_{2}$ loading and temperature were considered in addition to the uncertainty sources in the model equations during the uncertainty evaluation. The temperature accuracy of DMA 4500 and Physica MCR 101 Anton Paar are both specified as $\pm 0.03 \mathrm{~K}$. Considered standard uncertainties $u$ for the density measurements are $u(\alpha)= \pm 0.005\left(\mathrm{CO}_{2}\right.$ loading $\mathrm{mol} \mathrm{CO} 2 / \mathrm{mol}$ MEA), $u(w)= \pm 2 \times 10^{-4} \mathrm{~kg}$ (weight measurement), $u(p)= \pm 0.003$ (MEA purity), $u(T)= \pm 0.012 \mathrm{~K}$ (temperature) and $u(\mathrm{rep})= \pm 0.13 \mathrm{~kg} \cdot \mathrm{m}^{-3}$ (repeatability). The gradient $\partial \rho / \partial T$ of density against temperature was found as $0.73 \mathrm{~kg} \cdot \mathrm{m}^{-3} \cdot \mathrm{K}^{-1}$ and the corresponding uncertainty in $\rho$ that is $(\partial \rho / \partial T) u(T)$ was calculated as $\pm 0.009 \mathrm{~kg} \cdot \mathrm{m}^{-3}$. The gradient of density against $\mathrm{CO}_{2}$ loading, $\partial \rho / \partial \alpha$, was found as $334 \mathrm{~kg} \mathrm{~m}^{-3}$ and the corresponding uncertainty in $\rho,(\partial \rho / \partial \alpha) u(\alpha)$ was found as $\pm 1.67 \mathrm{~kg} \cdot \mathrm{m}^{-3}$. The standard combined uncertainty for density measurement $u(\rho)$ was found as $u(\rho)= \pm 3.90 \mathrm{~kg} \cdot \mathrm{m}^{-3}$. Accordingly the combined expanded uncertainty $U_{c}(\rho)$ for density of $\mathrm{CO}_{2}$-loaded aqueous MEA is $U_{c}(\rho)= \pm 7.80 \mathrm{~kg} \cdot \mathrm{m}^{-3}$ (level of confidence $=0.95$, where $k=2$ ).

The considered standard uncertainties $u$ for the viscosity measurements are $u(\alpha)= \pm 0.005\left(\mathrm{CO}_{2}\right.$ loading $\mathrm{mol} \mathrm{CO} 2 / \mathrm{mol} \mathrm{MEA}$ ), $u(w)= \pm 2 \times 10^{-4} \mathrm{~kg}$ (weight measurement), $u(p)= \pm 0.003$ (MEA purity), 
$u(T)= \pm 0.012 \mathrm{~K}$ (temperature) and $u(r e p)= \pm 0.008 \mathrm{mPa} \cdot \mathrm{s}$ (repeatability). The standard combined uncertainty for viscosity measurement $u(\eta)$ was found as $u(\eta)= \pm 0.018 \mathrm{mPa} \cdot \mathrm{s}$. Accordingly the combined expanded uncertainty $U_{c}$ is $U_{c}(\eta)= \pm 0.036 \mathrm{mPa} \cdot \mathrm{s}$ (level of confidence $=0.95$, where $k=2$ ).

\section{Results and Discussion}

This section discusses the density, viscosity and free energy of activation for viscous flow in aqueous and $\mathrm{CO}_{2}$-loaded aqueous MEA solutions. The correlations to represent density and viscosity data were evaluated using average absolute relative deviation and absolute maximum deviation (AARD and AMD) as given in Equations (1) and (2).

$$
\begin{gathered}
\operatorname{AARD}(\%)=\frac{100 \%}{N} \sum_{i=1}^{N}\left|\frac{Y_{i}^{E}-Y_{i}^{C}}{Y_{i}^{E}}\right| \\
A M D=\operatorname{MAX}\left|Y_{i}^{E}-Y_{i}^{C}\right|
\end{gathered}
$$

where $N, Y_{i}^{E}$ and $Y_{i}^{C}$ refer the number of data points, the measured property and calculated property respectively.

\subsection{Density of $\mathrm{MEA}(1)+\mathrm{H}_{2} \mathrm{O}$ (2) $+\mathrm{CO}_{2}$ (3) Mixtures}

Many approaches in density correlations are based on suggesting a Redlich-Kister polynomial to fit the excess volume properties of the mixture. One of the drawbacks of the excess volume approach using a Redlich-Kister polynomial to calculate density is the complexity of the correlation due to a high number of parameters. The density correlation proposed by Aronu, Hartono and Svendsen [14] as given by Equation (3) was used to fit the measured aqueous density data. The estimated parameters are presented in Table 2. The correlation was in good agreement with measured data with AARD = $0.12 \%$ for the $w_{1}$ range from 0.3 to 0.9 . The same parameters were used to fit the density of $\mathrm{CO}_{2}$-loaded solutions by introducing a function with new parameters for the temperature and $\mathrm{CO}_{2}$ mole fraction as illustrated in Equation (4).

Table 2. Correlation parameters for density of aqueous MEA.

\begin{tabular}{cccc}
\hline MEA $/ w_{1}$ & T/K & No. Points & Parameters \\
\hline & & & $k_{1}=683.5$ \\
$0.3-0.9$ & $293.15-363.15$ & \multirow{2}{*}{56} & $k_{2}=1.344 \times 10^{5}$ \\
& & & $k_{3}=-1.089 \times 10^{4}$ \\
& & & $k_{4}=145.2$ \\
& & $k_{5}=567.9$ \\
\hline & AARD (\%) & 0.12 \\
& AMD $\left(\mathrm{kg} \cdot \mathrm{m}^{-3}\right)$ & 3.45 \\
\hline
\end{tabular}

Correlation for the density of aqueous MEA:

$$
\rho=\left(k_{1}+\frac{k_{2} x_{2}}{T}\right) \exp \left(\frac{k_{3}}{T^{2}}+\frac{k_{4} x_{1}}{T}+k_{5}\left(\frac{x_{1}}{T}\right)^{2}\right)
$$

where $\rho, T, x_{1}, x_{2}$ and $k_{i}$ are density, temperature, mole fractions of $\mathrm{MEA}, \mathrm{H}_{2} \mathrm{O}$ of the aqueous mixture and estimated parameter vector.

The measured densities of aqueous MEA solutions are listed in Table 3. A comparison between correlations that are based on excess volume presented by Hartono, Mba and Svendsen [10] and Han, Jin, Eimer and Melaaen [1] with this work is shown in Figure 1. The accuracy of the correlation fit is acceptable compared to the literature $[1,10]$. The correlation deviates from measured density 
with AMD of $3.45 \mathrm{~kg} \cdot \mathrm{m}^{-3}$ at $w_{1}=0.8$ and $T=293.15 \mathrm{~K}$. This deviation is less than the measurement uncertainty reported in this study for aqueous MEA.

Table 3. Measured density $\rho / \mathrm{kg} \cdot \mathrm{m}^{-3}$ of aqueous MEA ${ }^{\mathrm{a}, \mathrm{b}, \mathrm{c}, \mathrm{d}, \mathrm{e}}$.

\begin{tabular}{|c|c|c|c|c|c|c|c|c|c|}
\hline \multirow{2}{*}{$w_{1}$} & \multirow{2}{*}{$x_{1}$} & \multicolumn{8}{|c|}{ Measured Density $\rho / \mathrm{kg} \cdot \mathrm{m}^{-3}$} \\
\hline & & $293.15 \mathrm{~K}$ & $303.15 \mathrm{~K}$ & $313.15 \mathrm{~K}$ & $323.15 \mathrm{~K}$ & $333.15 \mathrm{~K}$ & $343.15 \mathrm{~K}$ & $353.15 \mathrm{~K}$ & $363.15 \mathrm{~K}$ \\
\hline 0.3 & 0.1122 & $\begin{array}{c}1012.6 \\
1012.68^{d}\end{array}$ & $\begin{array}{c}1008.2^{2} \\
1008.4^{\mathrm{b}} \\
1008.31^{\mathrm{d}} \\
1008.2^{\mathrm{e}}\end{array}$ & $\begin{array}{c}1003.3^{\mathrm{b}} \\
1003.3^{\mathrm{b}} \\
1003.4^{\mathrm{c}} \\
1003.45^{\mathrm{d}} \\
1003.3^{\mathrm{e}}\end{array}$ & $\begin{array}{c}997.9^{\mathrm{b}} \\
998.1^{\mathrm{b}} \\
998.1^{\mathrm{c}} \\
998.7^{\mathrm{d}} \\
998.1^{\mathrm{e}}\end{array}$ & $\begin{array}{c}991.6 \\
992.3^{b} \\
992.23^{d} \\
992.3^{e}\end{array}$ & $\begin{array}{c}986.0^{\mathrm{b}} \\
986.1^{\mathrm{b}} \\
985.8^{\mathrm{c}} \\
985.6^{\mathrm{d}}\end{array}$ & $\begin{array}{c}979.4 \\
979.4^{\mathrm{b}} \\
979.4^{\mathrm{c}} \\
979.27^{\mathrm{d}}\end{array}$ & $\begin{array}{c}972.3^{\mathrm{b}} \\
972.5^{\mathrm{b}}\end{array}$ \\
\hline 0.4 & 0.1643 & 1018.4 & $\begin{array}{c}1013.3^{1} \\
1013.8^{\mathrm{b}} \\
1013.3^{\mathrm{e}}\end{array}$ & $\begin{array}{c}1007.8^{1} \\
1008.3^{\mathrm{b}} \\
1007.7^{\mathrm{c}} \\
1007.8^{\mathrm{e}}\end{array}$ & $\begin{array}{c}1001.8 \\
1002.3^{\mathrm{b}} \\
1001.8^{\mathrm{c}} \\
1002.1^{\mathrm{e}}\end{array}$ & $\begin{array}{c}995.5^{\mathrm{b}} \\
996.1^{\mathrm{b}} \\
995.7^{\mathrm{e}}\end{array}$ & $\begin{array}{c}988.9^{\mathrm{b}} \\
989.4^{\mathrm{b}} \\
988.9^{\mathrm{c}}\end{array}$ & $\begin{array}{c}981.9^{\mathrm{b}} \\
982.4^{\mathrm{b}} \\
981.9^{\mathrm{c}}\end{array}$ & $\begin{array}{c}974.6 \\
975.0^{\mathrm{b}}\end{array}$ \\
\hline 0.5 & 0.2278 & 1023.6 & $\begin{array}{c}1017.8 \\
1018.2^{b} \\
1017.8^{\mathrm{e}}\end{array}$ & $\begin{array}{c}1011.6 \\
1012.1^{b} \\
1011.7^{c} \\
1011.8^{e}\end{array}$ & $\begin{array}{c}1005.2^{2} \\
1005.6^{\mathrm{b}} \\
1005.3^{\mathrm{c}} \\
1005.4^{\mathrm{e}}\end{array}$ & $\begin{array}{c}998.4 \\
999.0^{\mathrm{b}} \\
998.7^{\mathrm{e}}\end{array}$ & $\begin{array}{c}991.4^{\mathrm{b}} \\
991.9^{\mathrm{b}} \\
991.5^{\mathrm{c}}\end{array}$ & $\begin{array}{c}984.1^{\mathrm{b}} \\
984.5^{\mathrm{b}} \\
984.2^{\mathrm{c}}\end{array}$ & $\begin{array}{c}976.4^{\mathrm{b}} \\
976.9^{\mathrm{b}}\end{array}$ \\
\hline 0.6 & 0.3067 & 1027.7 & $\begin{array}{c}1021.2 \\
1021.4^{\mathrm{b}} \\
1021.3^{\mathrm{e}}\end{array}$ & $\begin{array}{c}1014.5 \\
1014.7^{\mathrm{b}} \\
1014.6^{\mathrm{e}}\end{array}$ & $\begin{array}{c}1007.6 \\
1007.8^{\mathrm{b}} \\
1007.8^{\mathrm{e}}\end{array}$ & $\begin{array}{c}1000.4 \\
1000.7^{\mathrm{b}} \\
1000.6^{\mathrm{e}}\end{array}$ & $\begin{array}{c}993.0 \\
993.2^{\mathrm{b}}\end{array}$ & $\begin{array}{c}985.4 \\
985.6^{b}\end{array}$ & $\begin{array}{c}977.4^{b} \\
977.7^{\mathrm{b}}\end{array}$ \\
\hline 0.7 & 0.4077 & 1029.3 & $\begin{array}{c}1022.4^{1} \\
1022.8^{b} \\
1022.6^{e}\end{array}$ & $\begin{array}{c}1015.2^{2} \\
1015.7^{b} \\
1015.5^{c} \\
1015.5^{e}\end{array}$ & $\begin{array}{c}1007.9^{-} \\
1008.3^{\mathrm{b}} \\
1008.2^{\mathrm{c}} \\
1008.2^{\mathrm{e}}\end{array}$ & $\begin{array}{c}1000.4^{1} \\
1000.8^{b} \\
1000.6^{e}\end{array}$ & $\begin{array}{c}992.7^{\mathrm{b}} \\
993.1^{\mathrm{b}} \\
993.0^{\mathrm{c}}\end{array}$ & $\begin{array}{c}984.8^{\mathrm{b}} \\
985.2^{\mathrm{b}} \\
985.0^{\mathrm{c}}\end{array}$ & $\begin{array}{c}976.4 \\
977.1^{b}\end{array}$ \\
\hline 0.8 & 0.5412 & 1028.1 & $\begin{array}{c}1020.8 \\
1021.0^{b}\end{array}$ & $\begin{array}{c}1013.3 \\
1013.5^{b}\end{array}$ & $\begin{array}{c}1005.7 \\
1005.9^{b}\end{array}$ & $\begin{array}{c}997.9 \\
998.2^{\mathrm{b}}\end{array}$ & $\begin{array}{c}990.0 \\
990.2^{b}\end{array}$ & $\begin{array}{c}981.9 \\
982.1^{b}\end{array}$ & $\begin{array}{c}973.6 \\
973.9^{\mathrm{b}}\end{array}$ \\
\hline 0.9 & 0.7264 & 1023.5 & $\begin{array}{c}1015.8 \\
1016.2^{b}\end{array}$ & $\begin{array}{c}1008.1^{b} \\
1008.5^{b} \\
1008.4^{c}\end{array}$ & $\begin{array}{c}1000.3 \\
1000.6^{\mathrm{b}} \\
1000.6^{\mathrm{c}}\end{array}$ & $\begin{array}{c}992.4 \\
992.7^{b}\end{array}$ & $\begin{array}{c}984.3^{\mathrm{b}} \\
984.6^{\mathrm{b}} \\
984.6^{\mathrm{c}}\end{array}$ & $\begin{array}{c}976.1^{\mathrm{b}} \\
976.5^{\mathrm{b}} \\
976.4^{\mathrm{c}}\end{array}$ & $\begin{array}{c}967.8 \\
968.1^{b}\end{array}$ \\
\hline 1 & 1.0000 & 1015.9 & $\begin{array}{c}1008.1 \\
1008.0^{b}\end{array}$ & $\begin{array}{c}1000.1 \\
1000.0^{b} \\
1000.3^{c}\end{array}$ & $\begin{array}{c}992.1 \\
992.0^{\mathrm{b}} \\
992.3^{\mathrm{c}}\end{array}$ & $\begin{array}{c}984.0 \\
983.9^{\mathrm{b}}\end{array}$ & $\begin{array}{c}975.9^{\mathrm{b}} \\
975.8^{\mathrm{b}} \\
976.0^{\mathrm{c}}\end{array}$ & $\begin{array}{c}967.6^{\mathrm{b}} \\
967.5^{\mathrm{b}} \\
967.8^{\mathrm{c}}\end{array}$ & $\begin{array}{c}959.3 \\
959.2^{\mathrm{b}}\end{array}$ \\
\hline
\end{tabular}

a Standard uncertainties $u$ are $u(w)= \pm 2 \times 10^{-4} \mathrm{~kg}, u(p)= \pm 0.003, u(T)= \pm 0.012 \mathrm{~K}, u($ rep $)= \pm 0.13 \mathrm{~kg} \cdot \mathrm{m}^{-3}$. The combined expanded uncertainty $U_{c}$ is $U_{c}(\rho)= \pm 7.10 \mathrm{~kg} \cdot \mathrm{m}^{-3}$ (level of confidence $=0.95$, where $\left.k=2\right)$ ). ${ }^{b}$ Han, Jin, Eimer and Melaaen [1], ${ }^{c}$ Amundsen, Øi and Eimer [6], d Hartono, Mba and Svendsen [10], ${ }^{\mathrm{e}}$ Jayarathna, Weerasooriya, Dayarathna, Eimer and Melaaen [8].

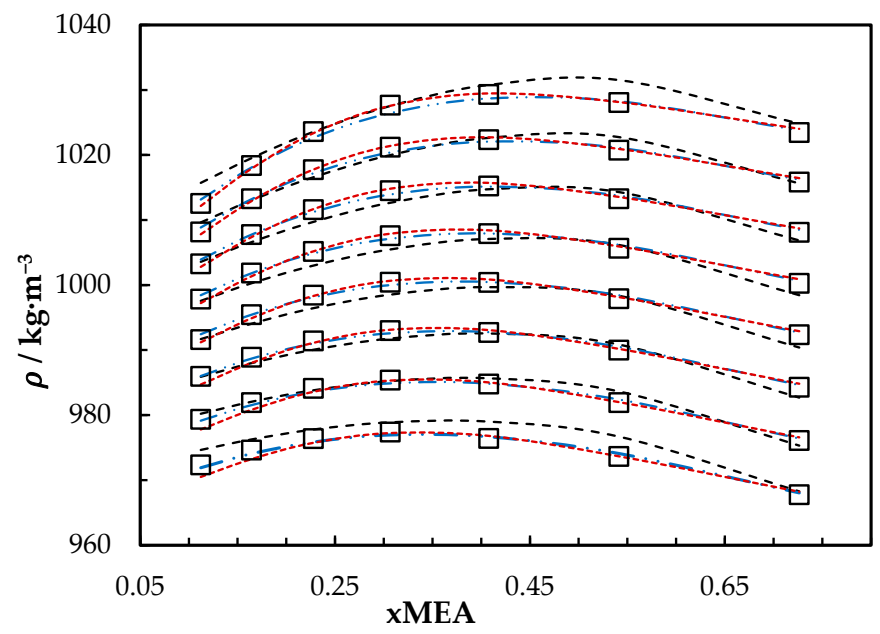

Figure 1. Density of aqueous MEA mixtures at different concentrations and temperatures $(293.15,303.15$, $313.15,323.15,333.15,343.15,353.15$ and 363.15) K. Data: from this work, ‘ $\square$ '. Correlation predictions: from this work, '- - -'; Hartono, Mba and Svendsen [10], '-..-'; Han, Jin, Eimer and Melaaen [1], '. . '. 
Correlation for the density of $\mathrm{CO}_{2}$-loaded aqueous MEA:

$$
\rho=\left(a_{1}+a_{2}(T)+a_{3}(T)^{2}+a_{4} x_{3}\right)\left(k_{1}+\frac{k_{2} x_{2}}{T}\right) \exp \left(\frac{k_{3}}{T^{2}}+\frac{k_{4} x_{1}}{T}+k_{5}\left(\frac{x_{1}}{T}\right)^{2}\right)
$$

The measured density of $\mathrm{CO}_{2}$-loaded aqueous MEA of $w_{1}=0.3,0.4$ and 0.5 solutions are shown in Table 4 and the correlation described in Equation (4) used to fit the data. At higher $\mathrm{CO}_{2}$ loadings $(\alpha>0.5)$, formation of air bubbles was noticed in the U-tube beyond temperatures of $323.15 \mathrm{~K}$ in DMA 4500. This increases the uncertainty of the density measurements. Accordingly, densities at temperatures up to $323.15 \mathrm{~K}$ are shown for the solutions with $w_{1}=0.3$ and 0.4 . The same was observed for the solution of $w_{1}=0.5$ with $\alpha=0.495$ at above $T=343.15 \mathrm{~K}$. Figure 2 shows the comparison of correlations proposed by Hartono, Mba and Svendsen [10], Han, Jin, Eimer and Melaaen [1] with this work for MEA solution of $w_{1}=0.3$. Measured densities at $w_{1}=0.4$ and 0.5 are given in Figures 3 and 4 with data from the literature. The correlation by Hartono, Mba and Svendsen [10] deviates positively from the measured data with AMD of $8.9 \mathrm{~kg} \cdot \mathrm{m}^{-3}$ while Han, Jin, Eimer and Melaaen [1] deviates negatively with AMD of $9.5 \mathrm{~kg} \cdot \mathrm{m}^{-3}$ at higher $\mathrm{CO}_{2}$ loadings. The required parameters of Equation (4) for the $\mathrm{CO}_{2}$-loaded solutions are listed in Table 5. The AMD from Equation (4) is lower than that from the other correlations.

Table 4. Measured density $\rho / \mathrm{kg} \cdot \mathrm{m}^{-3}$ of $\mathrm{CO}_{2}$-loaded $\left(\alpha / \mathrm{mol} \mathrm{CO}_{2} \cdot \mathrm{mol} \mathrm{MEA}^{-1}\right)$ aqueous MEA ${ }^{\text {a }}$.

\begin{tabular}{ccccccccc}
\hline \multirow{2}{*}{$\boldsymbol{x}_{3}$} & $\boldsymbol{\alpha}$ & \multicolumn{7}{c}{ Measured Density $\boldsymbol{\rho} / \mathbf{k g} \cdot \mathbf{m}^{-3}$} \\
\cline { 3 - 8 } & & $\mathbf{2 9 3 . 1 5} \mathbf{K}$ & $\mathbf{3 0 3 . 1 5} \mathbf{K}$ & $\mathbf{3 1 3 . 1 5} \mathbf{K}$ & $\mathbf{3 2 3 . 1 5} \mathbf{K}$ & $\mathbf{3 3 3 . 1 5} \mathbf{K}$ & $\mathbf{3 4 3 . 1 5} \mathbf{K}$ & $\mathbf{3 5 3 . 1 5} \mathbf{K}$ \\
\hline$w_{1}=0.3$ & & & & & & & \\
0.0000 & 0.000 & 1012.6 & 1008.2 & 1003.3 & 997.9 & 991.6 & 986.0 & 979.4 \\
0.0105 & 0.095 & 1032.0 & 1027.6 & 1022.8 & 1017.4 & 1011.6 & 1005.1 & 995.5 \\
0.0193 & 0.175 & 1052.5 & 1048.1 & 1043.3 & 1038.1 & 1032.4 & 1026.4 & 1020.1 \\
0.0355 & 0.328 & 1077.8 & 1073.4 & 1068.6 & 1063.4 & 1057.9 & 1052.0 & 1044.1 \\
0.0476 & 0.445 & 1103.3 & 1097.7 & 1092.8 & 1087.6 & 1082.1 & 1075.7 & 1069.3 \\
0.0574 & 0.543 & 1123.1 & 1118.4 & 1113.4 & 1107.9 & & & \\
\hline$w_{1}=0.4$ & & & & & & & & \\
0.0000 & 0.000 & 1018.4 & 1013.3 & 1007.8 & 1001.9 & 995.5 & 988.9 & 981.9 \\
0.0170 & 0.105 & 1045.6 & 1040.7 & 1035.3 & 1029.6 & 1023.6 & 1017.3 & 1010.6 \\
0.0341 & 0.215 & 1073.4 & 1068.5 & 1063.3 & 1057.8 & 1051.9 & 1045.8 & 1039.4 \\
0.0507 & 0.325 & 1102.0 & 1097.2 & 1092.0 & 1086.5 & 1080.8 & 1074.9 & 1068.6 \\
0.0669 & 0.436 & 1130.3 & 1125.4 & 1120.2 & 1114.7 & 1109.2 & 1103.2 & 1097.0 \\
0.0826 & 0.548 & 1155.5 & 1150.4 & 1145.1 & 1139.5 & & & \\
\hline$w_{1}=0.5$ & & & & & & & \\
0.0000 & 0.000 & 1023.6 & 1017.8 & 1011.6 & 1005.2 & 998.4 & 991.4 & 984.1 \\
0.0205 & 0.092 & 1052.3 & 1046.7 & 1040.9 & 1034.7 & 1028.3 & 1021.7 & 1014.8 \\
0.0406 & 0.186 & 1082.4 & 1077.0 & 1071.4 & 1065.5 & 1059.4 & 1053.0 & 1046.4 \\
0.0620 & 0.290 & 1112.7 & 1107.4 & 1101.9 & 1096.2 & 1090.3 & 1084.2 & 1077.9 \\
0.0825 & 0.395 & 1144.5 & 1139.2 & 1133.8 & 1128.3 & 1122.5 & 1116.6 & 1110.5 \\
0.1013 & 0.495 & 1175.7 & 1170.4 & 1165.0 & 1159.4 & 1153.6 & 1147.5 & \\
\hline 0
\end{tabular}

a Standard uncertainties $u$ are $u(\alpha)= \pm 0.005, u(w)= \pm 2 \times 10^{-4} \mathrm{~kg}, u(p)= \pm 0.003, u(T)= \pm 0.012 \mathrm{~K}, u($ rep $)= \pm 0.13 \mathrm{~kg} \cdot \mathrm{m}^{-3}$. The combined expanded uncertainty $U_{c}$ is $U_{c}(\rho)= \pm 7.80 \mathrm{~kg} \cdot \mathrm{m}^{-3}$ (level of confidence $=0.95$, where $k=2$ ).

Table 5. Density correlation parameters for $\mathrm{CO}_{2}$-loaded aqueous MEA.

\begin{tabular}{cccc}
\hline Parameters & $w_{1}=0.3$ & $w_{1}=0.4$ & $w_{1}=0.5$ \\
\hline$a_{1}$ & 0.6802 & 0.7731 & 0.7506 \\
$a_{2}$ & 0.001951 & 0.001354 & 0.001494 \\
$a_{3}$ & $-2.97 \times 10^{-6}$ & $-2.015 \times 10^{-6}$ & $-2.237 \times 10^{-6}$ \\
$a_{4}$ & 2.346 & 2.164 & 2.015 \\
\hline AARD $(\%)$ & 0.15 & 0.08 & 0.15 \\
AMD $\left(\mathrm{kg} \cdot \mathrm{m}^{-3}\right)$ & 4.2 & 2 & 3.8 \\
\hline
\end{tabular}




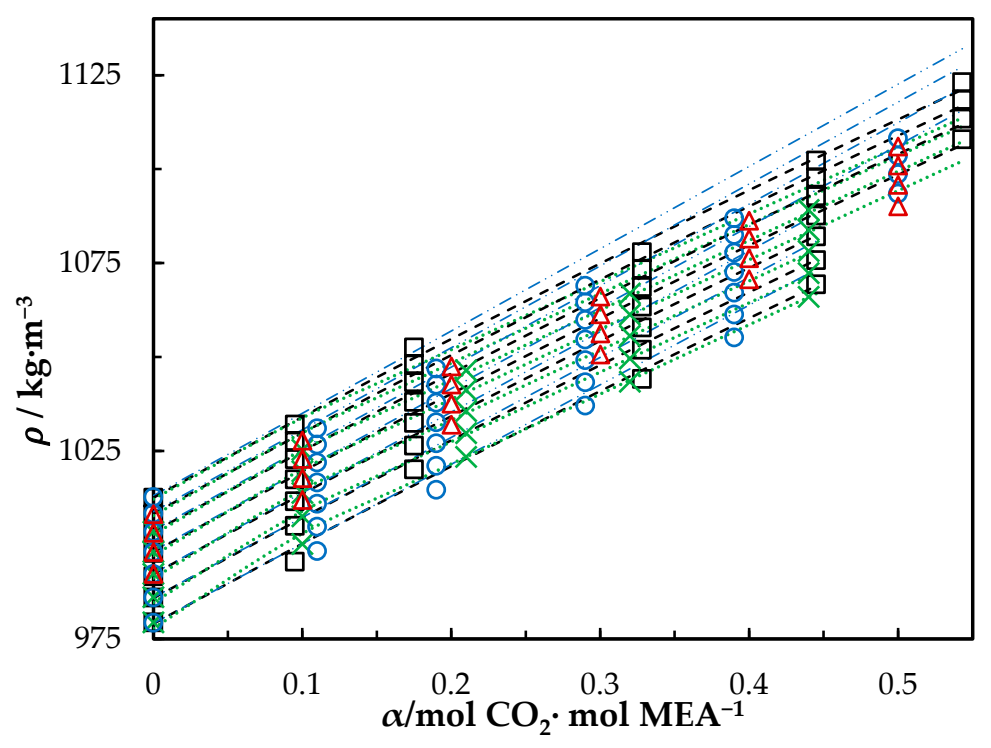

Figure 2. Density of $\mathrm{CO}_{2}$-loaded MEA $\left(w_{1}=0.3\right)$ solution at different $\mathrm{CO}_{2}$ loadings and temperatures $(293.15,303.15,313.15,323.15,333.15,343.15$ and 353.15) K. Data: from this work, ' $\square$ '; Hartono, Mba and Svendsen [10], 'O'; Han, Jin, Eimer and Melaaen [1], ' $x^{\prime}$; Jayarathna, Weerasooriya, Dayarathna, Eimer and Melaaen [8], ' $\Delta$ '. Correlation: from this work, '- - -'; Hartono, Mba and Svendsen [10], '..-- '; Han, Jin, Eimer and Melaaen [1], '..'.

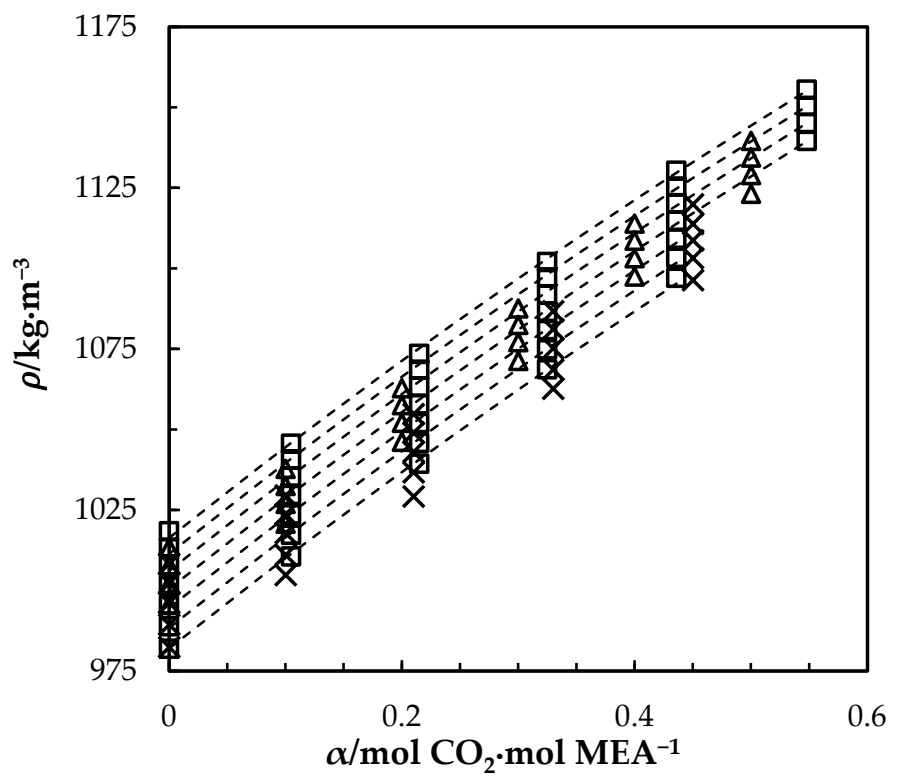

Figure 3. Density of $\mathrm{CO}_{2}$-loaded MEA $\left(w_{1}=0.4\right)$ solution at different $\mathrm{CO}_{2}$ loadings and temperatures $(293.15,303.15,313.15,323.15,333.15,343.15$ and 353.15) K. Data: from this work, ‘ $\square$ '; Han, Jin, Eimer and Melaaen [1], ' $x$ '; Jayarathna, Weerasooriya, Dayarathna, Eimer and Melaaen [8], ' $\triangle$ '. Correlation: from this work, '- - -'. 


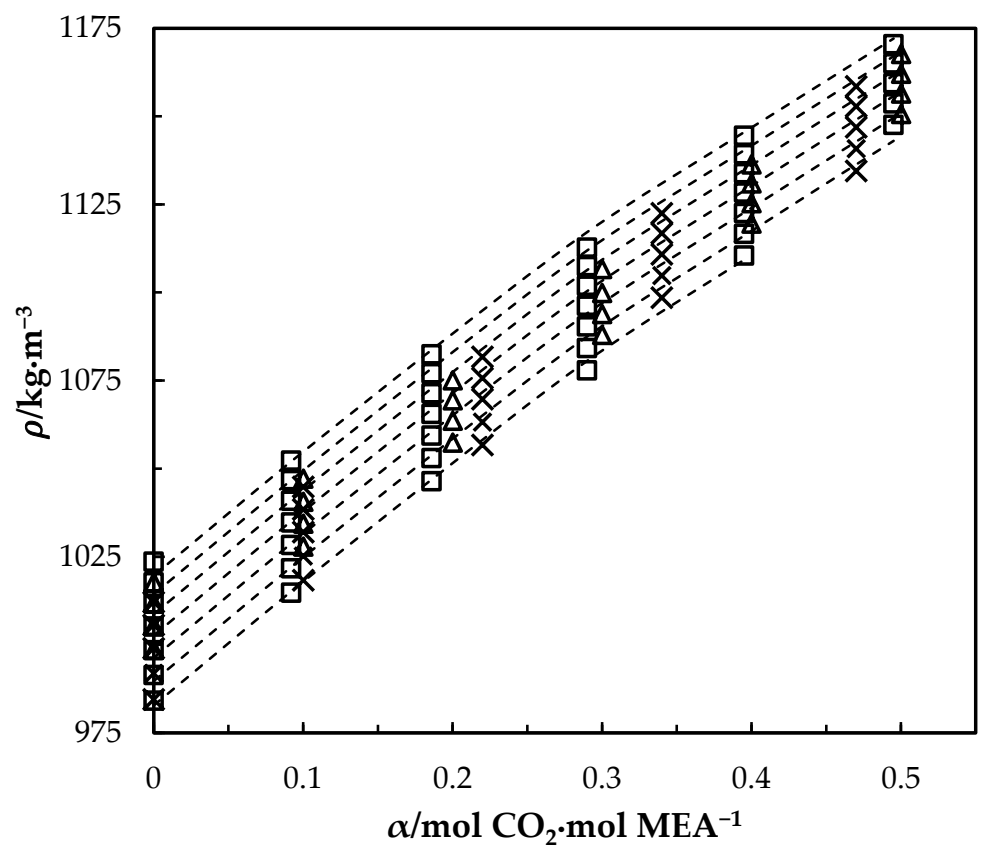

Figure 4. Density of $\mathrm{CO}_{2}$-loaded MEA $\left(w_{1}=0.5\right)$ solution at different $\mathrm{CO}_{2}$ loadings and temperatures $(293.15,303.15,313.15,323.15,333.15,343.15$ and 353.15) K. Data: from this work, ‘ $\square$ '; Han, Jin, Eimer and Melaaen [1], ' $x$ '; Jayarathna, Weerasooriya, Dayarathna, Eimer and Melaaen [8], ' $\triangle$ '. Correlation: from this work, '- - -'.

\subsection{Viscosity of $\mathrm{MEA}(1)+\mathrm{H}_{2} \mathrm{O}(2)+\mathrm{CO}_{2}$ (3) Mixtures}

The Eyring's viscosity model based on absolute rate theory is shown in Equation (5). Here, viscous flow is treated as a chemical reaction considering the elementary process as the motion of a single molecule from one equilibrium position to another over a potential energy barrier [21,22].

$$
\eta=\frac{h N_{A}}{V} \exp \left(\frac{\Delta G^{*}}{R T}\right)
$$

where $\eta, V, h, N_{A}, \Delta G^{*}, R$ and $T$ are dynamic viscosity, molar volume, Planck's constant, Avogadro's number, free energy of activation for viscous flow, universal gas constant and temperature respectively. For binary liquid mixtures, Equations (5) and (6) were adopted to derive Equation (7) to calculate excess free energy of activation for viscous flow $\Delta G^{E *}$.

$$
\begin{gathered}
\frac{\eta}{\eta_{\text {ideal }}}=\frac{V_{\text {ideal }}}{V} \exp \left(\frac{\Delta G^{E *}}{R T}\right) \\
\frac{\Delta G^{E *}}{R T}=\ln (\eta V)-\sum_{i=1}^{i=2} x_{i} \ln \left(\eta_{i} V_{i}^{0}\right)
\end{gathered}
$$

where $x_{i}, \eta_{i}$ and $V_{i}^{0}\left(i=1\right.$ for MEA and $i=2$ for $\left.\mathrm{H}_{2} \mathrm{O}\right)$ are the mole fraction of components in the mixture, dynamic viscosity and molar volume of pure liquids.

The $\Delta G^{E *}$ was evaluated via measured viscosity and density data of aqueous MEA for $w_{1}$ from 0.3 to 1 and MEA temperatures from $293.15 \mathrm{~K}$ to $363.15 \mathrm{~K}$. Viscosity and density of pure water for this study were taken from Korson, et al. [23] and Kestin, et al. [24]. A Redlich-Kister type correlation was used to fit the derived term $\Delta G^{E *} / R T$ and estimated parameters are given in Table 6 . The measured viscosities of aqueous MEA are tabulated with literature data in Table 7. Our previous work has reported viscosities of aqueous MEA from $w_{1}=0.3$ to $w_{1}=0.5$ in Karunarathne, et al. [25]. Figure 5 
shows the calculated and fitted $\Delta G^{E *}$ and Figure 6 compares the measured with calculated viscosities using the proposed correlation in this work and correlations suggested in the literature.

$$
\begin{gathered}
\frac{\Delta G^{E *}}{R T}=x_{1} x_{2} \sum_{i=0}^{i=2} C_{i}\left(1-2 x_{2}\right)^{i} \\
C_{i}=a_{i}+b_{i}(T)
\end{gathered}
$$

\begin{tabular}{|c|c|c|}
\hline$w_{1}$ & $T / K$ & Parameters \\
\hline $0-1$ & 298.15-363.15 & $\begin{array}{c}a_{0}=16.2 \\
b_{0}=-0.03473 \\
a_{1}=-4.853 \\
b_{1}=0.008315 \\
a_{2}=-6.433\end{array}$ \\
\hline \multicolumn{3}{|c|}{$R^{2}=0.998{ }^{b_{2}=0.02065}$} \\
\hline
\end{tabular}

Table 6. Parameters of the excess free energy of activation for viscous flow correlation.

\begin{tabular}{|c|c|c|c|c|c|c|c|c|c|}
\hline \multirow{2}{*}{$w_{1}$} & \multirow{2}{*}{$x_{1}$} & \multicolumn{8}{|c|}{ Measured Viscosity $\eta / \mathrm{mPa} \cdot \mathbf{s}$} \\
\hline & & $293.15 \mathrm{~K}$ & $303.15 \mathrm{~K}$ & $313.15 \mathrm{~K}$ & $323.15 \mathrm{~K}$ & $333.15 \mathrm{~K}$ & $343.15 \mathrm{~K}$ & $353.15 \mathrm{~K}$ & $363.15 \mathrm{~K}$ \\
\hline 0.3 & 0.1122 & $\begin{array}{c}2.836 \\
2.874^{b} \\
2.879^{b}\end{array}$ & $\begin{array}{c}2.109 \\
2.133^{b} \\
2.130^{b}\end{array}$ & $\begin{array}{c}1.628 \\
1.628^{b} \\
1.638^{b} \\
1.67^{c}\end{array}$ & $\begin{array}{c}1.290 \\
1.305^{b} \\
1.318^{b} \\
1.33^{c}\end{array}$ & $\begin{array}{c}1.046 \\
1.055^{b} \\
1.067^{b}\end{array}$ & $\begin{array}{c}0.866 \\
0.878^{b} \\
0.874^{b} \\
0.92^{c}\end{array}$ & $\begin{array}{c}0.740 \\
0.742^{\mathrm{b}} \\
0.740^{\mathrm{b}} \\
0.77^{\mathrm{c}}\end{array}$ & 0.687 \\
\hline 0.4 & 0.1643 & 4.285 & 3.080 & $\begin{array}{l}2.305^{\circ} \\
2.28^{c}\end{array}$ & $\begin{array}{l}1.782 \\
1.75^{\mathrm{c}}\end{array}$ & 1.417 & $\begin{array}{l}1.154 \\
1.14^{\mathrm{c}}\end{array}$ & $\begin{array}{l}0.960 \\
0.95^{c}\end{array}$ & 0.808 \\
\hline 0.5 & 0.2278 & 6.610 & $\begin{array}{l}4.580 \\
4.69^{\mathrm{d}}\end{array}$ & $\begin{array}{l}3.310 \\
3.39^{c} \\
3.37^{d}\end{array}$ & $\begin{array}{l}2.454 \\
2.54^{\mathrm{c}} \\
2.53^{\mathrm{d}}\end{array}$ & $\begin{array}{l}1.915 \\
1.94 \mathrm{~d}\end{array}$ & $\begin{array}{l}1.528 \\
1.57^{\mathrm{c}} \\
1.54^{\mathrm{d}}\end{array}$ & $\begin{array}{l}1.243 \\
1.28^{\mathrm{c}} \\
1.26^{\mathrm{d}}\end{array}$ & $\begin{array}{l}1.029 \\
1.05^{\mathrm{d}}\end{array}$ \\
\hline 0.6 & 0.3067 & 10.217 & $\begin{array}{l}6.769 \\
6.92^{d}\end{array}$ & $\begin{array}{l}4.736 \\
4.77^{d}\end{array}$ & $\begin{array}{l}3.444 \\
3.45^{\mathrm{d}}\end{array}$ & $\begin{array}{l}2.602 \\
2.62^{d}\end{array}$ & $\begin{array}{l}2.031 \\
2.04^{d}\end{array}$ & $\begin{array}{l}1.620 \\
1.62^{\mathrm{d}}\end{array}$ & $\begin{array}{l}1.319 \\
1.34^{\mathrm{d}}\end{array}$ \\
\hline 0.7 & 0.4077 & 15.348 & $\begin{array}{l}9.823 \\
9.89 \mathrm{~d}\end{array}$ & $\begin{array}{l}6.664 \\
6.96^{c} \\
6.69^{d}\end{array}$ & $\begin{array}{l}4.720 \\
4.94^{\mathrm{c}} \\
4.76^{\mathrm{d}}\end{array}$ & $\begin{array}{l}3.461 \\
3.49^{\mathrm{d}}\end{array}$ & $\begin{array}{l}2.615^{c} \\
2.79^{c} \\
2.63^{d}\end{array}$ & $\begin{array}{l}2.029 \\
2.18^{\mathrm{c}} \\
2.04^{\mathrm{d}}\end{array}$ & $\begin{array}{l}1.616 \\
1.63^{d}\end{array}$ \\
\hline 0.8 & 0.5412 & 20.521 & $\begin{array}{l}12.840 \\
13.38^{\mathrm{d}}\end{array}$ & $\begin{array}{l}8.534 \\
8.82^{d}\end{array}$ & $\begin{array}{l}5.937 \\
6.11^{\mathrm{d}}\end{array}$ & $\begin{array}{l}4.295 \\
4.41^{d}\end{array}$ & $\begin{array}{l}3.217 \\
3.26^{d}\end{array}$ & $\begin{array}{l}2.483 \\
2.49^{d}\end{array}$ & $\begin{array}{l}1.962 \\
1.97^{\mathrm{d}}\end{array}$ \\
\hline 0.9 & 0.7264 & 24.027 & $\begin{array}{c}14.963 \\
15.12^{d}\end{array}$ & $\begin{array}{c}9.879 \\
10.20^{\mathrm{c}} \\
9.95^{\mathrm{d}}\end{array}$ & $\begin{array}{l}6.829 \\
7.06^{\mathrm{c}} \\
6.88^{\mathrm{d}}\end{array}$ & $\begin{array}{l}4.936 \\
4.94^{\mathrm{d}}\end{array}$ & $\begin{array}{l}3.683 \\
3.81^{\mathrm{c}} \\
3.67^{\mathrm{d}}\end{array}$ & $\begin{array}{l}2.832^{\mathrm{c}} \\
2.93^{\mathrm{c}} \\
2.82^{\mathrm{d}}\end{array}$ & $\begin{array}{l}2.222 \\
2.23^{d}\end{array}$ \\
\hline 1 & 1.0000 & 23.376 & $\begin{array}{c}14.748 \\
14.77^{d}\end{array}$ & $\begin{array}{l}10.108 \\
9.61^{\mathrm{c}} \\
9.84^{\mathrm{d}}\end{array}$ & $\begin{array}{l}6.935 \\
6.72^{\mathrm{c}} \\
6.87^{\mathrm{d}}\end{array}$ & $\begin{array}{l}5.067 \\
4.98^{\mathrm{d}}\end{array}$ & $\begin{array}{l}3.834 \\
3.69^{c} \\
3.72^{d}\end{array}$ & $\begin{array}{l}2.974 \\
2.85^{\mathrm{c}} \\
2.85^{\mathrm{d}}\end{array}$ & $\begin{array}{l}2.364 \\
2.26^{\mathrm{d}}\end{array}$ \\
\hline
\end{tabular}

Table 7. Measured viscosity $\eta$ of aqueous MEA ${ }^{\mathrm{a}, \mathrm{b}, \mathrm{c}, \mathrm{d}}$.

${ }^{\text {a }}$ The pressure was maintained by $\mathrm{N}_{2}$ gas $(p=4$ bar) during the experiments. Standard uncertainties $u$ are $u(w)=$ $\pm 2 \times 10^{-4} \mathrm{~kg}, u(p)= \pm 0.003, u(T)= \pm 0.012 \mathrm{~K}, u(\mathrm{rep})= \pm 0.008 \mathrm{mPa} \cdot \mathrm{s}$. The combined expanded uncertainty $U_{c}$ is $U_{c}(\eta)= \pm 0.016 \mathrm{mPa} \cdot \mathrm{s}$ (level of confidence $=0.95$, where $k=2$ ). ${ }^{\mathrm{b}}$ Hartono, Mba and Svendsen [10], ${ }^{\mathrm{c}}$ Amundsen, Øi and Eimer [6], ${ }^{\mathrm{d}}$ Idris, et al. [26].

The viscosities from the correlation were in good agreement with measured data as shown in Figure 6. The proposed correlation was able to calculate viscosities with AARD 1.4\% and with AMD $0.79 \mathrm{mPa} \cdot \mathrm{s}$. Table 8 summarizes the AARD and AMD of different suggested correlations.

Figure 5 illustrates the variation of $\Delta G^{E *}$ over the whole range of concentrations at different temperatures. At a specific temperature, $\Delta G^{E *}$ increases with the increase of MEA concentration until it reaches a maximum at $x_{M E A}$ about 0.41 and then gradually decreases. The $\Delta G^{E *}$ decreases with the 
increase of temperature while composition for maximum $\Delta G^{E^{*}}$ is almost constant. A similar effect was observed for other aqueous amine mixtures [27,28].

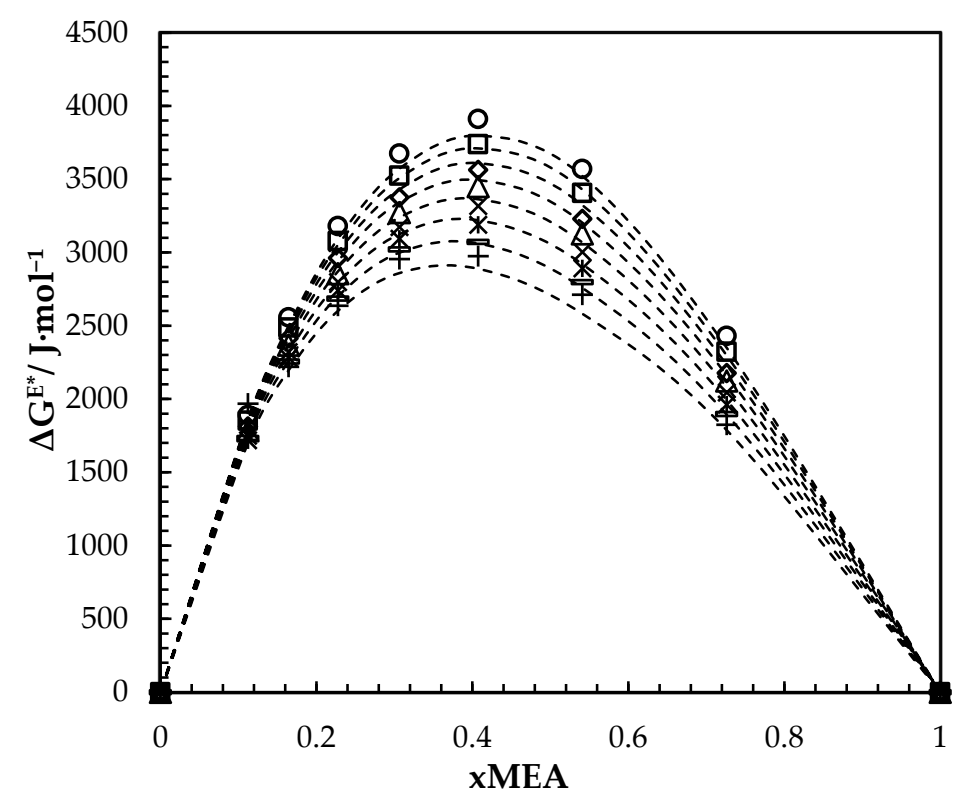

Figure 5. Calculated and fitted $\Delta G^{E^{*}}$ for aqueous MEA solutions at different concentrations and

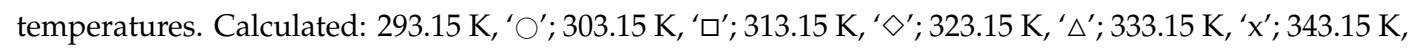
'ж'; 353.15 K, '-'; $363.15 \mathrm{~K},{ }^{\prime}+$ '. Correlation: '- - -'.

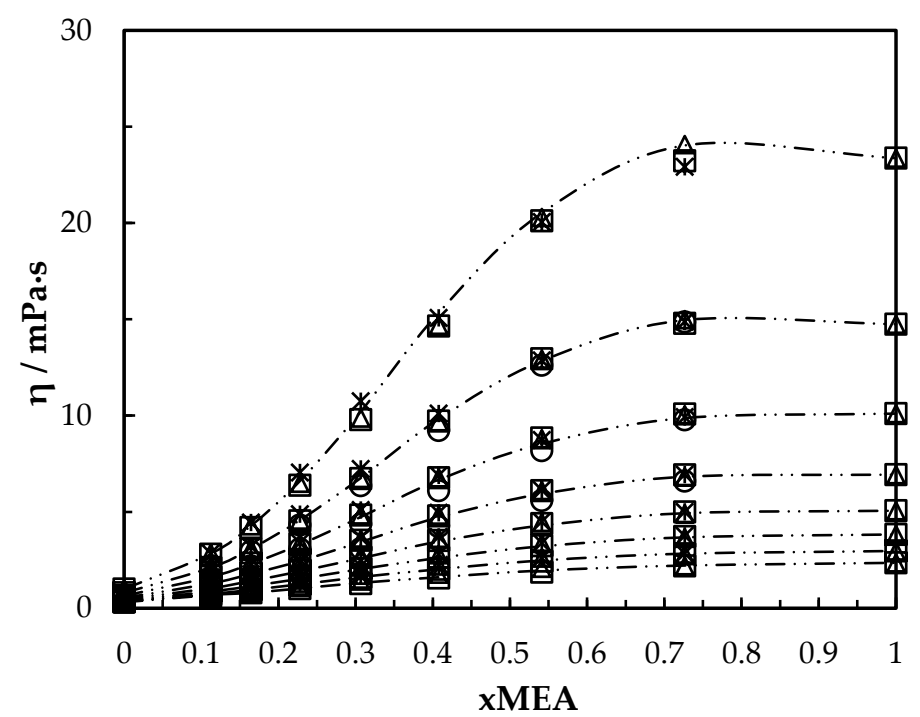

Figure 6. Viscosity of aqueous MEA solutions at different concentrations and temperatures (293.15, 303.15, 313.15, 323.15, 333.15, 343.15, 353.15, 363.15 K). Data: from this work, '-..-'. Correlation: from this work, ' $\square$ '; Hartono, Mba and Svendsen [10], ' $\triangle$ '; Arachchige, Aryal, Eimer and Melaaen [11], 'ж'; Islam, Islam and Yeasmin [17], ' $\bigcirc$ '.

Table 8. Average absolute relative deviations and absolute maximum deviation of different suggested correlations for viscosity of aqueous MEA solutions from $w_{1}=0$ to $w_{1}=1$ and $293.15-363.15 \mathrm{~K}$.

\begin{tabular}{cccc}
\hline Source (s) & No. Parameters & AARD (\%) & AMD (mPa.s) \\
\hline This work & 6 & 1.4 & 0.79 \\
Hartono, et al. [10] & 4 & 2.4 & 0.66 \\
Arachchige, et al. [11] & 7 & 3.5 & 1.1 \\
Islam, et al. [17] & 4 & 5.1 & 0.59 \\
\hline
\end{tabular}


The excess volume $V^{E}$ and excess viscosity $\eta^{E}$ of aqueous MEA was determined by Equations (10) and (11) to analyze the molecular interaction between MEA and $\mathrm{H}_{2} \mathrm{O}$.

$$
\begin{gathered}
V^{E}=V-\left(x_{1} V_{1}^{0}+x_{2} V_{2}^{0}\right) \\
\eta^{E}=\eta-\left(x_{1} \eta_{1}+x_{2} \eta_{2}\right)
\end{gathered}
$$

The $\Delta G^{E *}>0$ and $V^{E}<0$ for the considered MEA concentrations while $\eta^{E}$ is negative $(<0)$ for the water-rich region and gradually become positive $(>0)$ with the increase of MEA concentration. The $\Delta G^{E *}>0$ indicates that the viscosity of aqueous MEA solutions has greater viscosities than that of ideal mixtures [29]. The $V^{E}$ can be negative as a result of the chemical or specific interaction and the structural contribution due to the difference in shape and size [30]. According to Eyring's viscosity model, it can be argued that more energy is required to make necessary holes for molecules to jump in when they are closely packed. The sign of $\eta^{E}$ emphasizes strong specific interactions such as hydrogen bonding, which causes complex formations in the amine-rich region and weak interactions in the water-rich region [31].

The viscosity of $\mathrm{CO}_{2}$-loaded aqueous MEA solutions is given by Table 9 for $w_{1}=0.3,0,4$ and 0.5 under different $\mathrm{CO}_{2}$ loading in the temperature range from $293.15-353.15 \mathrm{~K}$. The measured viscosities at $w_{1}=0.3, w_{1}=0.4$ and 0.5 are shown in Figures 7-9 respectively with data from the literature. It was observed that the viscosity of solution increases with the increase of $\mathrm{CO}_{2}$ dissolved in the mixture for all three different MEA concentrations and it decreases with the increase of temperature. The $\Delta G^{*}$ was calculated for both $\mathrm{CO}_{2}$-loaded and $\mathrm{CO}_{2}$-unloaded solutions and the difference was considered to develop a correlation as shown in Equations (12) and (13) to predict the viscosity of $\mathrm{CO}_{2}$-loaded solutions.

Table 9. Measured viscosity of $\mathrm{CO}_{2}$-loaded $\left(\alpha / \mathrm{mol} \mathrm{CO}_{2}\right.$ mol·MEA $\left.{ }^{-1}\right)$ aqueous MEA ${ }^{\mathrm{a}}$.

\begin{tabular}{ccccccccc}
\hline \multirow{2}{*}{$x_{3}$} & $\alpha$ & \multicolumn{7}{c}{ Measured Viscosity $(\eta / \mathbf{m P a} \cdot \mathbf{s})$} \\
\cline { 3 - 9 } & & $\mathbf{2 9 3 . 1 5} \mathbf{K}$ & $\mathbf{3 0 3 . 1 5} \mathbf{K}$ & $\mathbf{3 1 3 . 1 5} \mathbf{K}$ & $\mathbf{3 2 3 . 1 5} \mathbf{K}$ & $\mathbf{3 3 3 . 1 5} \mathbf{K}$ & $\mathbf{3 4 3 . 1 5} \mathbf{K}$ & $\mathbf{3 5 3 . 1 5} \mathbf{K}$ \\
\hline$w_{1}=0.3$ & & & & & & & \\
0.0000 & 0.000 & 2.836 & 2.109 & 1.628 & 1.290 & 1.046 & 0.866 & 0.740 \\
0.0105 & 0.095 & 3.103 & 2.305 & 1.768 & 1.397 & 1.128 & 0.937 & 0.788 \\
0.0193 & 0.175 & 3.338 & 2.476 & 1.910 & 1.511 & 1.228 & 1.021 & 0.865 \\
0.0355 & 0.328 & 3.730 & 2.764 & 2.138 & 1.699 & 1.384 & 1.152 & 0.977 \\
0.0476 & 0.445 & 4.164 & 3.105 & 2.403 & 1.913 & 1.562 & 1.308 & 1.118 \\
0.0574 & 0.543 & 4.515 & 3.360 & 2.602 & 2.064 & 1.680 & 1.403 & 1.191 \\
\hline$w_{1}=0.4$ & & & & & & & & \\
0.0000 & 0.000 & 4.285 & 3.080 & 2.305 & 1.782 & 1.417 & 1.154 & 0.960 \\
0.0170 & 0.105 & 4.793 & 3.423 & 2.567 & 1.985 & 1.590 & 1.302 & 1.090 \\
0.0341 & 0.215 & 5.524 & 3.944 & 2.968 & 2.308 & 1.851 & 1.526 & 1.286 \\
0.0507 & 0.325 & 6.496 & 4.655 & 3.502 & 2.726 & 2.198 & 1.813 & 1.524 \\
0.0669 & 0.436 & 7.639 & 5.442 & 4.084 & 3.177 & 2.556 & 2.111 & 1.781 \\
0.0826 & 0.548 & 8.820 & 6.203 & 4.614 & 3.544 & 2.821 & 2.302 & 1.917 \\
\hline$w_{1}=0.5$ & & & & & & & & \\
0.0000 & 0.000 & 6.610 & 4.580 & 3.310 & 2.454 & 1.915 & 1.528 & 1.243 \\
0.0205 & 0.092 & 7.859 & 5.378 & 3.926 & 2.955 & 2.303 & 1.838 & 1.493 \\
0.0406 & 0.186 & 9.518 & 6.529 & 4.756 & 3.594 & 2.813 & 2.269 & 1.866 \\
0.0620 & 0.290 & 11.611 & 7.904 & 5.710 & 4.291 & 3.328 & 2.667 & 2.190 \\
0.0825 & 0.395 & 14.854 & 10.073 & 7.247 & 5.422 & 4.227 & 3.409 & 2.809 \\
0.1013 & 0.495 & 19.348 & 12.841 & 9.068 & 6.678 & 5.169 & 4.118 & 3.365 \\
\hline
\end{tabular}

a The pressure was maintained by $\mathrm{N}_{2}$ gas $(p=4$ bar) during the experiments. Standard uncertainties $u$ are $u(\alpha)=$ $\pm 0.005, u(w)= \pm 2 \times 10^{-4} \mathrm{~kg}, u(p)= \pm 0.003, u(T)= \pm 0.012 \mathrm{~K}, u($ rep $)= \pm 0.008 \mathrm{mPa} \cdot \mathrm{s}$. 


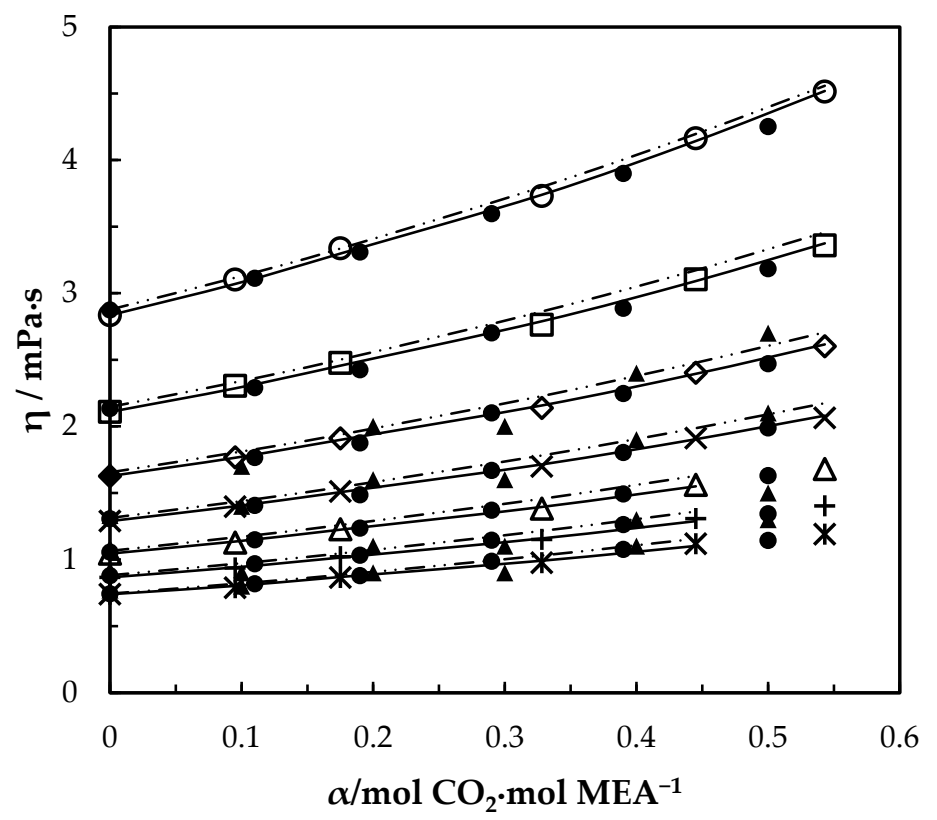

Figure 7. Viscosity of $\mathrm{CO}_{2}$-loaded aqueous MEA $\left(w_{1}=0.3\right)$ solutions at different $\mathrm{CO}_{2}$ loadings and

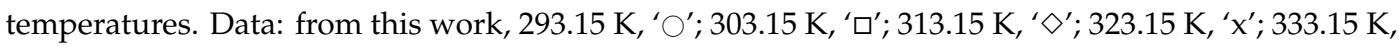

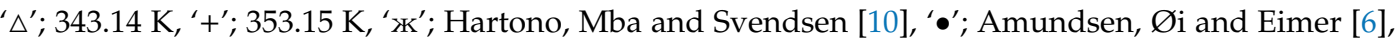

'৯'. Correlation: from this work, '- - -'; Hartono, Mba and Svendsen [10], '-..-'.

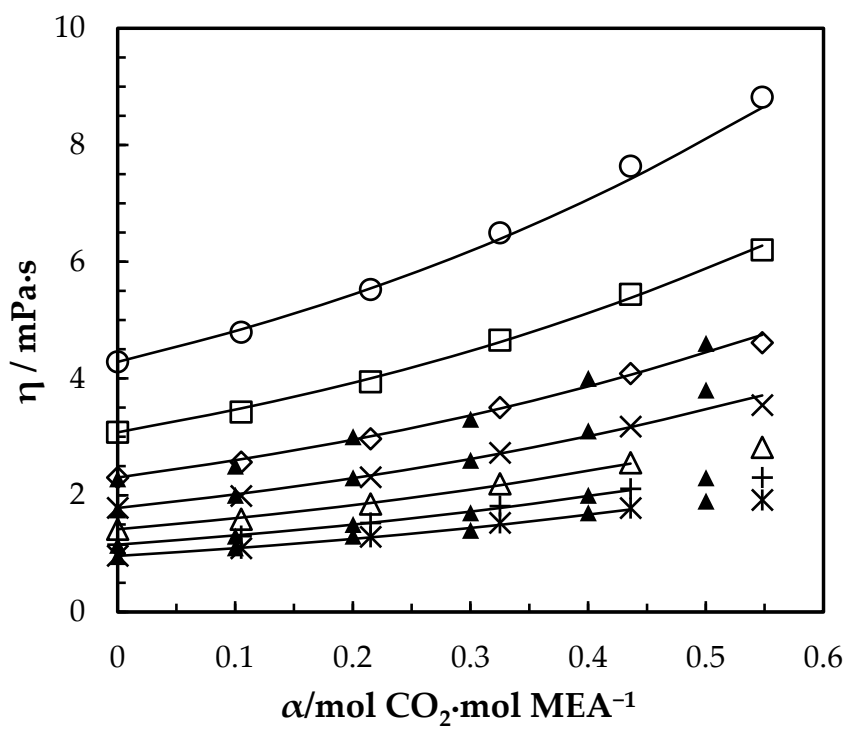

Figure 8. Viscosity of $\mathrm{CO}_{2}$-loaded aqueous MEA $\left(w_{1}=0.4\right)$ solutions at different $\mathrm{CO}_{2}$ loadings and

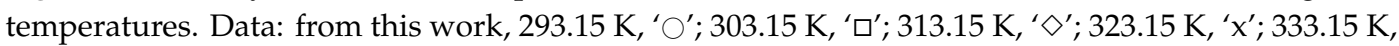

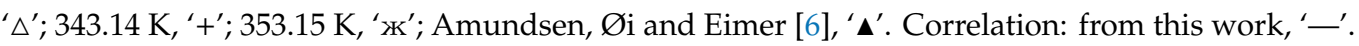

The combined expanded uncertainty $U_{c}$ is $U_{c}(\eta)= \pm 0.036 \mathrm{mPa} \cdot \mathrm{s}$ (level of confidence $=0.95$, where $k=2$ ).

$$
\begin{gathered}
\ln (V \eta)_{\mathrm{CO} 2 \text { loaded }}=\ln (V \eta)_{\text {unloaded }}+f\left(x_{3}, T\right) \\
f\left(x_{3}, T\right)=x_{3}\left(d_{1}+d_{2} T+d_{3} x_{3}\right)
\end{gathered}
$$




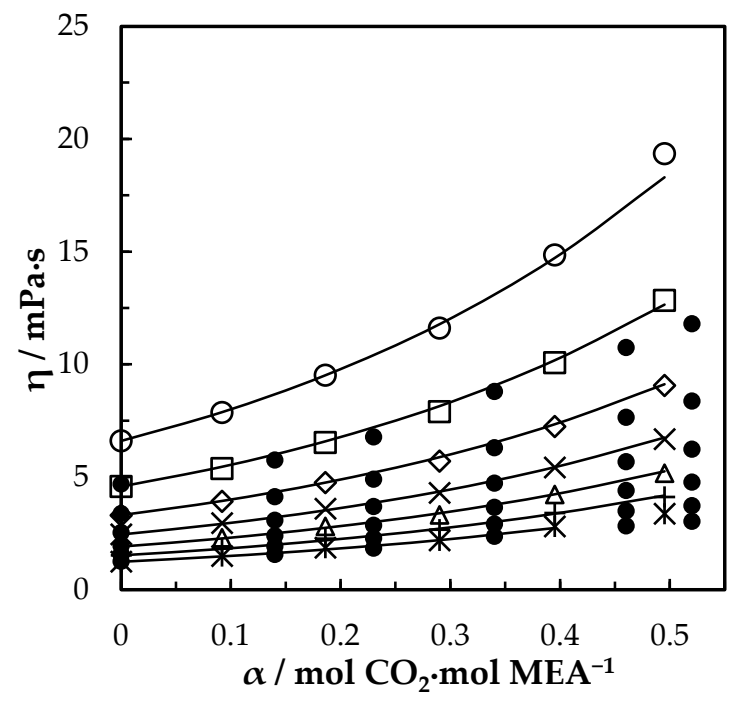

Figure 9. Viscosity of $\mathrm{CO}_{2}$-loaded aqueous MEA $\left(w_{1}=0.5\right)$ solutions at different $\mathrm{CO}_{2}$ loadings and

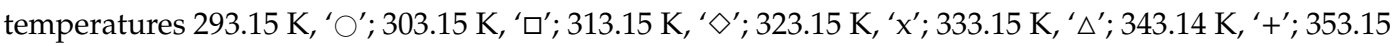
$\mathrm{K}$, ' $\mathrm{K}^{\prime}$; Idris, Kummamuru and Eimer [26], ' $\bullet$ '. Correlation: from this work, '-'.

The calculated AARD shows that the predicted and measured viscosities are in good agreement and parameters for the correlation are given in Table 10. The molar volume of $\mathrm{CO}_{2}$-loaded aqueous MEA solutions was calculated using the mole fraction of dissolved $\mathrm{CO}_{2}$ that was determined via $\mathrm{CO}_{2}$ loading analysis. In a real solution, $\mathrm{CO}_{2}$ reacts with MEA to form carbamate and bicarbonate ions and the solution becomes an electrolyte. Here it is assumed as unreacted and molar volumes were calculated using Equation (14) [32]. This approach was taken to represent dissolved $\mathrm{CO}_{2}$ in aqueous MEA $[7,10,26]$ and used in the viscosity correlation by Hartono, Mba and Svendsen [10].

$$
V_{\text {loaded }}=\frac{\sum_{1}^{3} x_{i} M_{i}}{\rho_{\text {loaded }}}
$$

Table 10. Parameters of viscosity correlation for $\mathrm{CO}_{2}$-loaded solutions.

\begin{tabular}{ccccc}
\hline T/K & Parameters & $\boldsymbol{w}_{1}=0.3$ & $\boldsymbol{w}_{1}=0.4$ & $\boldsymbol{w}_{\mathbf{1}}=\mathbf{0 . 5}$ \\
\hline \multirow{3}{*}{$298.15-343.15$} & $d_{1}$ & 4.536 & 2.554 & 8.533 \\
& $d_{2}$ & 0.006765 & 0.01205 & -0.0037 \\
& $d_{3}$ & 12.08 & 19.46 & 17.79 \\
\hline AARD (\%) & 0.58 & 1.13 & 1.25 \\
AMD (mPa.s) & 0.03 & 0.22 & 1.04 \\
\hline
\end{tabular}

The variations of $\Delta G^{*}$ with $\mathrm{CO}_{2}$ loading and temperature are shown in Figure 10a-c. For $\mathrm{CO}_{2}$ loaded solutions, $\Delta G^{*}$ increases with the increase of dissolved $\mathrm{CO}_{2}$ while it decreases with temperature. The amount of ions present in the solution due to the formation of carbamate and bicarbonate increases with the $\mathrm{CO}_{2}$ loading, which results in higher ionic strength as discussed by Matin, et al. [33]. At higher ionic strengths, ions can create an ionic field that attract water molecules to form clusters, which leads to higher viscosity. The increase of $\Delta G^{*}$ implies the increase of potential barrier for the molecule transfer. The molecular interactions among the molecules in $\mathrm{CO}_{2}$-loaded solutions may enhance the strength of energy barrier more than that of unloaded solutions. The correlation given in Equation (15) was proposed to fit $\Delta G^{*}$ for the $\mathrm{CO}_{2}$-loaded aqueous MEA of $w_{1}=0.3,0,4$ and 0.5. On the other hand, since the Eyring's viscosity model is based on the motion of individual molecules from one equilibrium position to another; it does not explain the effect of hydrogen bond network on the bulk viscosity of the various $\mathrm{CO}_{2}$-loaded aqueous [34] MEA solutions. Further, the model has molar volume as a parameter 
that needs to be known to calculate the viscosity. This can be done by using calculated molar volume from density data measured under the same conditions or from a correlation.

$$
\Delta G_{\mathrm{CO} 2 \text { loaded }}^{*}=\Delta G_{\text {unloaded }}^{*}+x_{3} R T\left(d_{1}+d_{2} T+d_{3} x_{3}\right)
$$

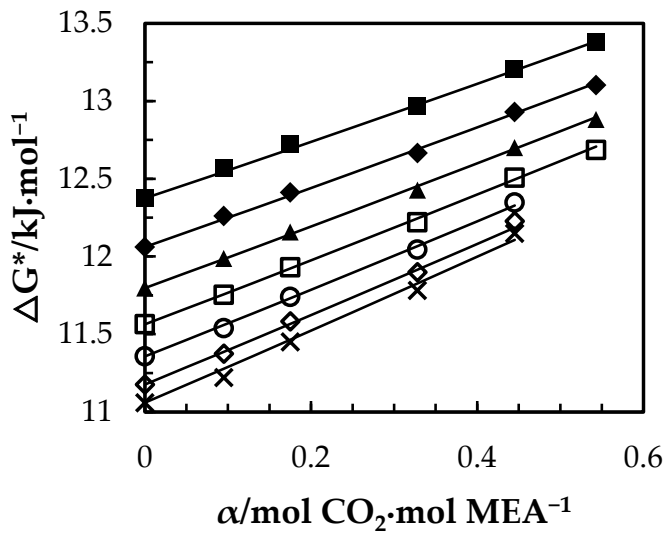

(a) $w_{1}=0.3$

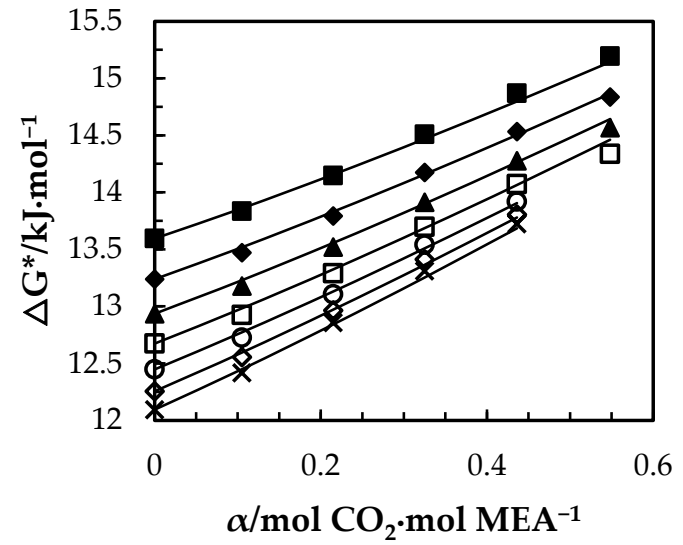

(b) $w_{1}=0.4$

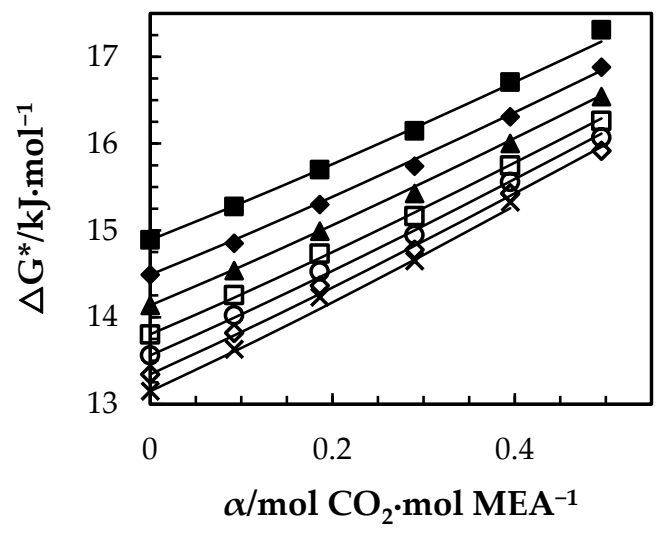

(c) $w_{1}=0.5$

Figure 10. Variation of free energy of activation for viscous flow of $\mathrm{CO}_{2}$-loaded aqueous MEA: (a) $w_{1}=$ $0.3,(\mathbf{b}) w_{1}=0.4,(\mathbf{c}) w_{1}=0.5$ solutions at different $\mathrm{CO}_{2}$ loadings and temperatures of $T=293.15 \mathrm{~K}$, ' $\mathbf{m}$;

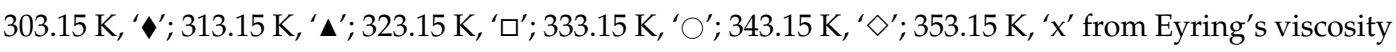
model. '-' from correlation in Equation (15).

The relationship between $\operatorname{Rln}\left(\eta V /\left(h N_{A}\right)\right)$ vs. $1 / T$ gives information about activation parameters in which enthalpy of activation for viscous flow $\Delta H^{*}$ is given by the gradient and entropy of activation for viscous flow $\Delta S^{*}$ is given by the intercept of the curve under different mole fractions of the components. The $\Delta G^{*}, \Delta H^{*}$ and $\Delta S^{*}$ are connected through the equation $\Delta G^{*}=\Delta H^{*}-T \Delta S^{*}$. Accordingly, Eyring's viscosity model is given as follows.

$$
\eta=\frac{h N_{A}}{V} \exp \left(\frac{\Delta H^{*}}{R T}-\frac{\Delta S^{*}}{R}\right)
$$

Tables 11 and 12 list the calculated $\Delta G^{*}$ directly from Eyring's viscosity model, and $\Delta H^{*}$ and $\Delta S^{*}$ from the relation shown in Equation (16). It is observed that $\Delta G^{*}, \Delta H^{*}$ and $\Delta S^{*}$ are positive for all considered mixtures while $\Delta H^{*}$ is greater than $T \Delta S^{*}$. This reveals that the influence of enthalpy of activation to the free energy of activation is greater than entropy of activation for viscous flow. Further, 
this work shows how $\Delta G^{*}$ can be regarded as a parameter to regress and also can be regarded as a parameter with a physical meaning.

Table 11. Free energy of activation for viscous flow $\Delta G^{*} / \mathrm{kJ} \cdot \mathrm{mol}^{-1}$ for $\mathrm{CO}_{2}$-loaded aqueous MEA.

\begin{tabular}{|c|c|c|c|c|c|c|c|c|}
\hline \multicolumn{2}{|c|}{$\mathrm{T} / \mathrm{K}$} & 293.15 & 303.15 & 313.15 & 323.15 & 333.15 & 343.15 & 353.15 \\
\hline$w_{1}$ & $x_{3}$ & \multicolumn{7}{|c|}{ Free Energy $\Delta G^{*} / \mathrm{kJ} \cdot \mathrm{mol}^{-1}$} \\
\hline \multirow{6}{*}{0.3} & 0.0000 & 12.375 & 12.062 & 11.798 & 11.564 & 11.358 & 11.177 & 11.060 \\
\hline & 0.0105 & 12.571 & 12.262 & 11.988 & 11.753 & 11.539 & 11.376 & 11.222 \\
\hline & 0.0193 & 12.721 & 12.413 & 12.159 & 11.931 & 11.740 & 11.583 & 11.451 \\
\hline & 0.0355 & 12.970 & 12.667 & 12.428 & 12.220 & 12.044 & 11.900 & 11.783 \\
\hline & 0.0476 & 13.210 & 12.931 & 12.702 & 12.507 & 12.347 & 12.228 & 12.150 \\
\hline & 0.0574 & 13.382 & 13.105 & 12.883 & 12.685 & & & \\
\hline \multirow{6}{*}{0.4} & 0.0000 & 13.595 & 13.240 & 12.936 & 12.674 & 12.448 & 12.255 & 12.094 \\
\hline & 0.0170 & 13.835 & 13.471 & 13.179 & 12.924 & 12.726 & 12.556 & 12.419 \\
\hline & 0.0341 & 14.148 & 13.793 & 13.521 & 13.291 & 13.105 & 12.965 & 12.860 \\
\hline & 0.0507 & 14.509 & 14.175 & 13.914 & 13.698 & 13.540 & 13.413 & 13.312 \\
\hline & 0.0669 & 14.870 & 14.534 & 14.278 & 14.072 & 13.919 & 13.807 & 13.725 \\
\hline & 0.0826 & 15.194 & 14.837 & 14.568 & 14.337 & & & \\
\hline \multirow{6}{*}{0.5} & 0.0000 & 14.891 & 14.489 & 14.137 & 13.802 & 13.560 & 13.344 & 13.148 \\
\hline & 0.0205 & 15.275 & 14.853 & 14.538 & 14.255 & 14.022 & 13.819 & 13.631 \\
\hline & 0.0406 & 15.701 & 15.299 & 14.992 & 14.733 & 14.526 & 14.366 & 14.230 \\
\hline & 0.0620 & 16.147 & 15.741 & 15.426 & 15.165 & 14.945 & 14.779 & 14.648 \\
\hline & 0.0825 & 16.707 & 16.309 & 16.002 & 15.747 & 15.559 & 15.427 & 15.325 \\
\hline & 0.1013 & 17.311 & 16.879 & 16.543 & 16.262 & 16.069 & 15.918 & \\
\hline
\end{tabular}

Table 12. Free energy of activation for viscous flow $\Delta G^{*} / \mathrm{kJ}^{\mathrm{mol}}{ }^{-1}$ for $\mathrm{CO}_{2}$-loaded aqueous MEA.

\begin{tabular}{cccc}
\hline $\boldsymbol{w}_{1}$ & $\boldsymbol{x}_{3}$ & $\boldsymbol{\Delta} \boldsymbol{H}^{*} / \mathbf{k J} \cdot \mathbf{m o l}^{-\mathbf{1}}$ & $\boldsymbol{\Delta} \boldsymbol{S}^{*} / \mathbf{J} \cdot(\mathbf{m o l} \cdot \mathbf{K})^{-1}$ \\
\hline \multirow{4}{*}{0.3} & 0.0000 & 18.834 & 22.301 \\
& 0.0105 & 19.150 & 22.696 \\
& 0.0193 & 18.902 & 21.360 \\
& 0.0355 & 18.716 & 19.895 \\
& 0.0476 & 18.400 & 18.003 \\
& 0.0574 & 20.173 & 23.234 \\
\hline \multirow{4}{*}{0.4} & 0.0000 & 20.897 & 25.215 \\
& 0.0170 & 20.688 & 23.742 \\
& 0.0341 & 20.377 & 21.642 \\
& 0.0507 & 20.266 & 20.026 \\
& 0.0669 & 20.379 & 19.209 \\
& 0.0826 & 23.540 & 28.578 \\
\hline \multirow{6}{*}{0.5} & 0.0000 & 23391 & 29.339 \\
& 0.0205 & 23147 & 27.247 \\
& 0.0406 & 22773 & 24.566 \\
& 0.0620 & 23389 & 25.142 \\
& 0.0825 & 23381 & 23.248 \\
& 0.1013 & 25441 & 28.114 \\
\hline
\end{tabular}

\section{Conclusions}

Densities and viscosities of MEA (1) $+\mathrm{H}_{2} \mathrm{O}$ (2) mixtures have been measured for the mass fraction $w_{1}$ from 0.3 to 1 and temperatures in the range $273.15 \mathrm{~K}$ to $363.15 \mathrm{~K}$. The density data were correlated using the correlation proposed by Aronu, Hartono and Svendsen for $w_{1}$ from 0.3 to 0.9 . The accuracy of the measured density with correlation predictions are acceptable as the AARD is $0.12 \%$ and AMD is $3.45 \mathrm{~kg} \cdot \mathrm{m}^{-3}$. The viscosity data were correlated using a Redlich-Kister type polynomial fitted to the excess free energy of activation for viscous flow $\Delta G^{E *}$ obtained via the Eyring's viscosity model for 
the $w_{1}$ from 0 to 1 and temperatures in a range from $273.15 \mathrm{~K}$ to $363.15 \mathrm{~K}$. The developed correlation was able to represent the measured viscosities with AARD $=1.4 \%$ and AMD $=0.79 \mathrm{mPa} \cdot \mathrm{s}$, which is acceptable in engineering calculations.

The densities of $\mathrm{CO}_{2}$-loaded aqueous MEA solutions were measured at temperatures ranging from $293.15 \mathrm{~K}$ to $353.15 \mathrm{~K}$ for $w_{1}$ of $0.3,0.4$ and 0.5 . Density of $\mathrm{CO}_{2}$-loaded solutions increases with the $\mathrm{CO}_{2}$ loading and decreases with temperature. The density correlation proposed by Aronu, Hartono and Svendsen was modified to correlate the density data. The AMD between correlated and experimental densities are $4.2 \mathrm{~kg} \cdot \mathrm{m}^{-3}, 2 \mathrm{~kg} \cdot \mathrm{m}^{-3}$ and $4.5 \mathrm{~kg} \cdot \mathrm{m}^{-3}$ for $\mathrm{CO}_{2}$-loaded solutions with $w_{1}$ of $0.3,0.4$ and 0.5 respectively.

The viscosities of $\mathrm{CO}_{2}$-loaded aqueous MEA solutions were measured at temperatures ranging from $293.15 \mathrm{~K}$ to $353.15 \mathrm{~K}$ for $w_{1}$ of $0.3,0.4$ and 0.5 . As $\mathrm{CO}_{2}$ loading increased, the viscosity increased and the viscosity decreased with the increase of temperature. A correlation was proposed for the free energy of activation for viscous flow using $\mathrm{CO}_{2}$ mole fraction and temperature to correlate viscosity data. The AMD between correlated and experimental viscosities are $0.03 \mathrm{mPa} \cdot \mathrm{s}, 0.22 \mathrm{mPa} \cdot \mathrm{s}$ and $1.04 \mathrm{mPa} \cdot \mathrm{s}$ for $\mathrm{CO}_{2}$-loaded solutions with $w_{1}$ of $0.3,0.4$ and 0.5 respectively. The proposed correlation is recommended to use in engineering calculations.

Author Contributions: Supervision, L.E.Ø. and D.A.E.; Writing-original draft, S.S.K. All authors have read and agreed to the published version of the manuscript.

Funding: This work was funded by the Ministry of Education and Research of the Norwegian Government.

Conflicts of Interest: The authors declare no conflict of interest.

\section{References}

1. Han, J.; Jin, J.; Eimer, D.A.; Melaaen, M.C. Density of water (1) + Monoethanolamine (2) + CO2 (3) from (298.15 to 413.15) K and surface tension of water (1) + Monoethanolamine (2) from (303.15 to 333.15) K. J. Chem. Eng. Data 2012, 57, 1095-1103. [CrossRef]

2. Nwaoha, C.; Saiwan, C.; Supap, T.; Idem, R.; Tontiwachwuthikul, P.; Rongwong, W.; Al-Marri, M.J.; Benamor, A. Carbon dioxide (CO2) capture performance of aqueoustri-solvent blends containing 2-amino-2-methyl-1-propanol (AMP) and methyldiethanolamine (MDEA) promoted by diethylenetriamine (DETA). Int. J. Greenh. Gas Control 2016, 53, 292-304. [CrossRef]

3. Kidnay, A.J.; Parrish, W.R. Fundamentals of Natural Gas Processing; Taylor \& Francis Group: Boca Raton, FL, USA, 2006.

4. Maham, Y.; Teng, T.T.; Hepler, L.G.; Mather, A.E. Densities, excess molar volumes, and partial molar volumes for binary mixtures of Water with Monoethanolamine, Diethnolamine, and Triethanolamine from 25 to $80^{\circ} \mathrm{C}$. J. Solut. Chem. 1994, 23, 195-205. [CrossRef]

5. Yang, F.; Wang, X.; Wang, W.; Liu, Z. Densities and excess properties of primary amines in alcoholic solutions. J. Chem. Eng. Data 2013, 58, 785-791. [CrossRef]

6. Amundsen, T.G.; Øi, L.E.; Eimer, D.A. Density and viscosity of monoethanolamine + water + carbon dioxide from $(25 \text { to } 80)^{\circ} \mathrm{C}$. J. Chem. Eng. Data 2009, 54, 3096-3100. [CrossRef]

7. Jayarathna, S.A.; Jayarathna, C.K.; Kottage, D.A.; Dayarathna, S.; Eimer, D.A.; Melaaen, M.C. Density and surface tension measurement of partially carbonated aqueous monoethanolamine solutions. J. Chem. Eng. Data 2013, 58, 343-348. [CrossRef]

8. Jayarathna, S.; Weerasooriya, A.; Dayarathna, S.; Eimer, D.A.; Melaaen, M.C. Densities and surface tensions of $\mathrm{CO} 2$ loaded aqueous monoethanolamine solution with $\mathrm{r}=(0.2$ to 0.7$)$ at $\mathrm{T}=(303.15$ to 333.15$) \mathrm{K}$. J. Chem. Eng. Data 2013, 58, 986-992. [CrossRef]

9. Weiland, R.H.; Dingman, J.C.; Cronin, D.B.; Browning, G.J. Density and viscosity of some partially carbonated aqueous alkanolamine solutions and their blends. J. Chem. Eng. Data 1998, 43, 378-382. [CrossRef]

10. Hartono, A.; Mba, E.O.; Svendsen, H.F. Physical properties of partially $\mathrm{CO} 2$ loaded aqueous monoethanolamine (MEA). J. Chem. Eng. Data 2014, 59, 1808-1816. [CrossRef]

11. Arachchige, U.S.P.R.; Aryal, N.; Eimer, D.A.; Melaaen, M.C. Viscosities of pure and aqueous solutions of Monoethanolamine (MEA), Diethanolamine (DEA), and N-Methyldiethanolamine (MDEA). In Proceedings of the Annual Transactions of the Nordic Rheology Society, Copenhagen, Demark, 12-14 June 2013. 
12. Hsu, C.-H.; Li, M.-H. Viscosities of Aqueous Blended Amines. J. Chem. Eng. Data 1997, 42, 714-720. [CrossRef]

13. Versteeg, G.F.; Van Swaaij, W.P.M. Solubility and diffusivity of acid gases (carbon dioxide, nitrous oxide) in aqueous alkanolamine solutions. J. Chem. Eng. Data 1988, 33, 29-34. [CrossRef]

14. Aronu, U.E.; Hartono, A.; Svendsen, H.F. Density, viscosity, and N2O solubility of aqueous amino acid salt and amine amino acid salt solutions. J. Chem. Thermodyn. 2012, 45, 90-99. [CrossRef]

15. McAllister, R.A. The viscosity of liquid mixtures. AIChE. J. 1960, 6, 427-431. [CrossRef]

16. Redlich, O.; Kister, A.T. Algebraic representation of thermodynamic properties and the classification of solutions. Ind. Eng. Chem. 1948, 40, 345-348. [CrossRef]

17. Islam, M.N.; Islam, M.M.; Yeasmin, M.N. Viscosity of aqueous solution of 2-methoxyethanol, 2-ethoxyethanol, and ethanolamine. J. Chem. Thermodyn. 2004, 36, 889-893. [CrossRef]

18. Eyring, H. Viscosity, Plasticity, and Diffusion as example of absolute reaction rates. J. Chem. Phys. 1936, 4, 283-291. [CrossRef]

19. JCGM. Evaluation of measurement data-Supplement 1 to the Guide to the Expression of Uncertainty In Measurement-Propagation of Distributions Using a Monte Carlo Method. In JCGM 101: 2008; JCGM: Sevres, France, 2008.

20. Ellison, S.L.R.; Williams, A. Eurachem/CITAC Guide: Quantifying Uncertainty in Analytical Measurement, 3rd ed.; 2012; Available online: http://www.eurachem.org (accessed on 15 November 2019).

21. Nhaesi, A.H. A Study of the Predictive Models for the Viscosity of Multi-Component Liquid Regular Solutions. Ph.D. Thesis, University of Windsor, Windsor, UK, 1998. Available online: https://core.ac.uk/ download/pdf/72774384.pdf (accessed on 15 November 2019).

22. Macías-Salinas, R.; Aquino-Olivos, M.A.; García-Sánchez, F. Viscosity modelling of reservoir fluids over wide temperature and pressure ranges. Chem. Eng. Trans. 2013, 32, 1573-1578. [CrossRef]

23. Korson, L.; Hansen, W.D.; Millero, F.J. Viscosity of water at various temperatures. J. Phys. Chem. 1969, 73, 34-39. [CrossRef]

24. Kestin, J.; Sokolov, M.; Wakeham, W.A. Viscosity of liquid water in the range $-8^{\circ} \mathrm{C}$ to $150{ }^{\circ} \mathrm{C}$. J. Phys. Chem. Ref. Data 1978, 7, 941-948. [CrossRef]

25. Karunarathne, S.S.; Eimer, D.A.; Øi, L.E. Evaluation of systematic error and uncertainty of viscosity measurements of mixtures of monoethanol amine and water in coaxial cylinder rheometers. Int. J. Model. Optim. 2018, 8, 260-265. [CrossRef]

26. Idris, Z.; Kummamuru, N.B.; Eimer, D.A. Viscosity measurement of unloaded and CO2-loaded aqueous monoethanolamine at higher concentrations. J. Mol. Liq. 2017, 243, 638-645. [CrossRef]

27. Hartono, A.; Svendsen, H.F. Density, viscosity, and excess properties of aqueous solution of diethylenetriamine (DETA). J. Chem. Thermodyn. 2009, 41, 973-979. [CrossRef]

28. Maham, Y.; Liew, C.N.; Mather, A.E. Viscosities and Excess Properties of Aqueous Solutions of Ethanolamines from 25 to $80^{\circ} \mathrm{C}$. J. Solut. Chem. 2002, 31, 743-756. [CrossRef]

29. Heric, E.L.; Brewer, J.G. Viscosity of some binary liquid nonelectrolyte mixtures. J. Chem. Eng. Data 1967, 12, 574-583. [CrossRef]

30. Mahajan, A.R.; Mirgane, S.R. Excess molar volumes and viscosities for the binary mixtures of n-Octane, n-Decane, n-Dodecane, and n-Tetradecane with Octan-2-ol at 298.15 K. J. Thermodyn. 2013, 2013, 1-11. [CrossRef]

31. Idris, Z.; Kummamuru, N.B.; Eimer, D.A. Viscosity measurement and correlation of unloaded and CO2 loaded 3-Amino-1-propanol solution. J. Chem. Eng. Data 2018, 63, 1454-1459. [CrossRef]

32. Stec, M.; Spietz, T.; Wieclaw-Solny, L.; Tatarczuk, A.; Wilk, A.; Sobolewski, A. Density of unloaded and CO2-loaded aqueous solutions of piperazine and 2-amino-2-methyl-1-propanol and their mixtures from 293.15 to 333.15 K. Phys. Chem. Liq. 2015, 54, 475-486. [CrossRef]

33. Matin, N.S.; Remias, J.E.; Liu, K. Application of electrolyte-NRTL model for prediction of the viscosity of carbon dioxide loaded aqueous amine solutions Ind. Eng. Chem. Res 2013, 52, 16979-16984. [CrossRef]

34. Perticaroli, S.; Mostofian, B.; Ehlers, G.; Neuefeind, J.C.; Diallo, S.O.; Stanley, C.B.; Daemen, L.; Egami, T.; Katsaras, J.; Cheng, X.; et al. Structural relaxation, viscosity, and network connectivity in a hydrogen bonding liquid. Phys. Chem. Chem. Phys. 2017, 19, 25859-25869. [CrossRef]

(C) 2020 by the authors. Licensee MDPI, Basel, Switzerland. This article is an open access article distributed under the terms and conditions of the Creative Commons Attribution (CC BY) license (http://creativecommons.org/licenses/by/4.0/). 